\title{
Magnetic fields of the W4 superbubble
}

\author{
X. Y. Gao ${ }^{1,2}$, W. Reich ${ }^{2}$, P. Reich ${ }^{2}$, J. L. Han ${ }^{1}$, and R. Kothes ${ }^{3}$ \\ ${ }^{1}$ National Astronomical Observatories, Chinese Academy of Sciences, Jia-20 Datun Road, Chaoyang District, 100012 Beijing, \\ PR China \\ e-mail: bearwards@gmail.com \\ 2 Max-Planck-Institut für Radioastronomie, Auf dem Hügel 69, 53121 Bonn, Germany \\ ${ }^{3}$ National Research Council of Canada, Dominion Radio Astrophysical Observatory, PO Box 248, Penticton BC, V2A 6J9, Canada \\ Received 10 September 2014 / Accepted 14 March 2015
}

\begin{abstract}
Context. Superbubbles and supershells are the channels for transferring mass and energy from the Galactic disk to the halo. Magnetic fields are believed to play a vital role in their evolution.

Aims. We study the radio continuum and polarized emission properties of the W4 superbubble to determine its magnetic field strength. Methods. New sensitive radio continuum observations were made at $\lambda 6 \mathrm{~cm}, \lambda 11 \mathrm{~cm}$, and $\lambda 21 \mathrm{~cm}$. The total intensity measurements were used to derive the radio spectrum of the W4 superbubble. The linear polarization data were analysed to determine the magnetic field properties within the bubble shells.

Results. The observations show a multi-shell structure of the W4 superbubble. A flat radio continuum spectrum that stems from optically thin thermal emission is derived from $1.4 \mathrm{GHz}$ to $4.8 \mathrm{GHz}$. By fitting a passive Faraday screen model and considering the filling factor $f_{n_{\mathrm{e}}}$, we obtain the thermal electron density $n_{\mathrm{e}}=1.0 / \sqrt{f_{n_{\mathrm{e}}}}( \pm 5 \%) \mathrm{cm}^{-3}$ and the strength of the line-of-sight component of the magnetic field $B_{/ /}=-5.0 / \sqrt{f_{n_{\mathrm{e}}}}( \pm 10 \%) \mu \mathrm{G}$ (i.e. pointing away from us) within the western shell of the W4 superbubble. When the known tilted geometry of the W4 superbubble is considered, the total magnetic field $B_{\text {tot }}$ in its western shell is greater than $12 \mu \mathrm{G}$. The electron density and the magnetic field are lower and weaker in the high-latitude parts of the superbubble. The rotation measure is found to be positive in the eastern shell but negative in the western shell of the W4 superbubble, which is consistent with the case where the magnetic field in the Perseus arm is lifted up from the plane towards high latitudes.

Conclusions. The magnetic field strength and the electron density we derived for the W4 superbubble are important parameters for evolution models of superbubbles breaking out of the Galactic plane.
\end{abstract}

Key words. ISM: magnetic fields - radio continuum: ISM - ISM: individual objects: W4 superbubble

\section{Introduction}

A large number of superbubbles and supershells have been found and discussed within our own Galaxy (e.g. Heiles 1979; Hu 1981; Heiles 1984; Koo et al. 1992; Maciejewski et al. 1996; Uyanıker et al. 2001; McClure-Griffiths et al. 2000, 2006; Pidopryhora et al. 2007) and in external galaxies (e.g. Meaburn 1980; Graham \& Lawrie 1982; Brinks \& Bajaja 1986; Deul \& den Hartog 1990; Kim et al. 1998). They are created either by energetic stellar winds from OB star associations, by multiple supernova explosions, or by a combination. Besides their identification by shell-like $\mathrm{H}$ I emission, superbubbles and supershells were also traced by soft X-ray (e.g. Cash et al. 1980) and $\mathrm{H} \alpha$ emission (e.g. Reynolds \& Ogden 1979). Superbubbles powered by sufficient energy will break out of the Galactic plane (Tomisaka \& Ikeuchi 1986; Mac Low \& McCray 1988; Mac Low et al. 1989) and form vertical "chimneys" in the interstellar medium (e.g. the Stockert chimney, Müller et al. 1987). A general scenario was proposed by Norman \& Ikeuchi (1989) that chimneys play an important role in the interaction between the Galactic disk and halo by carrying up mass, energy, momentum, and magnetic flux. Therefore it is of interest to understand the properties of the superbubbles and their evolution processes. However, so far only a few superbubbles and chimneys have been resolved well and the magnetic field strengths measured (e.g. Vallée 1993).
The W4 superbubble in the Perseus arm is one of the few superbubbles that extend over several degrees and that have been also previously resolved well in observations. It was first identified by Normandeau et al. (1996) from H I observations with the synthesis telescope of the Dominion Radio Astrophysical Observatory (DRAO; Landecker et al. 2000). A cone-shaped cavity was revealed to open upwards from its powering source, the young open star cluster OCl 352, which includes nine O-type stars. The lower part of the cavity is bounded by the well-known Galactic H II region W4. Evidence was found for an energetic outflow towards high positive latitudes, because $\mathrm{H}$ I streams were seen to point away from OCl 352. Because of the open conical shape viewed in the neutral gas, the entire structure was first named "W4 chimney". Follow-up H $\alpha$ observations (Dennison et al. 1997) showed that the ionized gas is detected in the periphery of the H I cavity and revealed an enclosed superbubble rather than a chimney, which is sealed at about $b=7^{\circ}$. As shown by West et al. (2007), the appearance of this huge structure seen from the $1.4 \mathrm{GHz}$ radio continuum emission also resembles a closed bubble. We therefore refer to it as "W4 superbubble" in this work. Basu et al. (1999) explained the morphological difference between a chimney and a superbubble by the penetration of UV photons to the higher latitude regions. Based on the study of infrared and radio continuum data, Terebey et al. (2003) find a large leakage of UV photons from $\mathrm{OCl} 352$. This leakage was indirectly confirmed by the discovery of an even larger 
Table 1. Observational parameters.

\begin{tabular}{lrrr}
\hline \multicolumn{1}{c}{ Data } & Urumqi $\lambda 6 \mathrm{~cm}$ & Effelsberg $\lambda 11 \mathrm{~cm}$ & EMLS $\lambda 21 \mathrm{~cm}$ \\
\hline Frequency [MHz] & $4800 / 4963$ & 2639 & 1400 \\
Bandwidth [MHz] & $600 / 295$ & 80 & 20 \\
HPBW ['] & 9.5 & 4.3 & 9.35 \\
Number of coverages & $6(\mathrm{~B})+4(\mathrm{~L})$ & $6(\mathrm{~B})+6(\mathrm{~L})$ & $1(\mathrm{~B})+1(\mathrm{~L})$ \\
Integration time $[\mathrm{s}]$ & 7.5 & 6 & 2 \\
rms $(I / U, Q)\left[\mathrm{mK} T_{\mathrm{b}}\right]$ & $0.7 / 0.4$ & $2.0 / 1.5$ & $15 / 8$ \\
\hline Calibrator & $3 \mathrm{C} 286$ & $3 \mathrm{C} 286$ & $3 \mathrm{C} 286$ \\
Flux density $[\mathrm{Jy}]$ & 7.5 & 11.5 & 14.4 \\
Polarization percentage & $11.3 \%$ & $9.9 \%$ & $9.3 \%$ \\
Polarization angle $\left[{ }^{\circ}\right]$ & 33 & 33 & 32 \\
\hline
\end{tabular}

high-latitude $\mathrm{H} \alpha$ loop on top of the W4 superbubble extending up to $b=30^{\circ}$ (Reynolds et al. 2001).

The distance to the W4 superbubble was taken as $2.2 \mathrm{kpc}$ in the work of Normandeau et al. (1996), Dennison et al. (1997), Reynolds et al. (2001), and Terebey et al. (2003), while 2.35 kpc was used by Basu et al. (1999) and West et al. (2007). Through measuring the trigonometric parallax of the methanol maser, $\mathrm{Xu}$ et al. (2006) got $1.95 \mathrm{kpc}$ for the distance of the $\mathrm{W} 3 \mathrm{OH}$ region. Considering a probable association between the $\mathrm{H}$ II regions W3 and W4 that gives another constraint on the distance of the W4 superbubble, we adopt the median value of $2.2 \mathrm{kpc}$ in the following analysis.

The age of the W4 superbubble has not been determined with high precision. Dennison et al. (1997) estimated an age between 6.4 and 9.6 Myr, while Basu et al. (1999) derived a lower value of about 2.5 Myr. According to the formation of the large high-latitude $\mathrm{H} \alpha$ loop found on top of the W4 superbubble, Reynolds et al. (2001) argue that the formation of the W4 superbubble is not a single event, but rather caused by sequential star formation. Age estimates, however, are inevitably affected by the presence and strength of the magnetic fields. Numerical simulations have indicated that the magnetic field has a significant impact on the evolution of superbubbles (e.g. Tomisaka 1990; Ferrière et al. 1991; Tomisaka 1998). The expansion of superbubbles differs along and perpendicular to the field lines and results in an elongated shape (de Avillez \& Breitschwerdt 2005; Stil et al. 2009). Komljenovic et al. (1999) have suggested that magnetic fields of a few $\mu \mathrm{G}$ must exist in the shells of the W4 superbubble to maintain its highly collimated shape and prevent its fragmentation from Rayleigh-Taylor instability. The strength of the line-of-sight component of the magnetic fields within the shells of the W4 superbubble was first estimated by West et al. (2007) based on the polarization data collected by the DRAO synthesis telescope. They determined a field strength between $3 \mu \mathrm{G}$ and $5 \mu \mathrm{G}$ by analysing lines through the $\mathrm{W} 4$ supperbubble shell separated by $5^{\prime}$. No short-spacing information was included in their data, which is, however, required to correctly interpret polarization structures resulting from Faraday rotation in the interstellar medium (e.g. Reich 2006; Sun et al. 2007; Landecker et al. 2010). We discuss the influence of polarized large-scale emission on this result in Sect. 4.2.

This work presents a study of the radio spectrum and the magnetic fields within the shells of the W4 superbubble based on new sensitive multi-frequency radio continuum and polarization observations including zero spacings. We describe the data sets we used in Sect. 2 and present all observational results and their analysis in Sect. 3. In Sect. 4, we use a Faraday screen model to derive the magnetic field strengths in the W4 superbubble. We discuss and summarize the results in Sect. 5.

\section{Data}

New sensitive radio continuum and linear polarization observations of the W4 superbubble have been made at $\lambda 6 \mathrm{~cm}$ with the Urumqi 25-m radio telescope of Xinjiang Astronomical Observatories, Chinese Academy of Sciences, and at $\lambda 11 \mathrm{~cm}$ with the Effelsberg 100-m radio telescope of the Max-PlanckInstitut für Radioastronomie, Germany. Total intensity and polarization data at $\lambda 21 \mathrm{~cm}$ were taken from an unpublished section of the Effelsberg Medium Latitude Survey (EMLS; Uyanıker et al. 1998, 1999; Reich et al. 2004). The Stockert $\lambda 21 \mathrm{~cm}$ total intensity northern sky survey (Reich 1982; Reich \& Reich 1986) and the DRAO 26-m single-dish polarization survey (Wolleben et al. 2006) provided the missing large-scale intensities and the absolute zero levels. We also added high-resolution $\lambda 21 \mathrm{~cm}$ data observed with the DRAO synthesis telescope by West et al. (2007) and Landecker et al. (2010) to the large-scale corrected EMLS maps.

Technical details of each observation, such as the central frequency, angular resolution, and sensitivity, are listed in Table 1 . The bandwidth depolarization (e.g. Crawford \& Tiffany 2007) for the single-channel observations by the Urumqi and Effelsberg telescopes was found to be negligible $(<1 \%)$ for all the three wavelengths, even for a rotation measure (RM) as large as $300 \mathrm{rad} \mathrm{m}^{-2}$.

\subsection{Urumqi $\lambda 6 \mathrm{~cm}$ data}

The $\lambda 6 \mathrm{~cm}$ total intensity and linear polarization data were obtained between 2007 and 2009 during the conduction of the Sino-German $\lambda 6 \mathrm{~cm}$ polarization survey of the Galactic plane (Sun et al. 2007; Gao et al. 2010; Sun et al. 2011; Xiao et al. 2011), using the same observational and reduction methods. The $\lambda 6 \mathrm{~cm}$ observations had two modes - the broad-band mode with a central frequency of $4800 \mathrm{MHz}$ and a bandwidth of $600 \mathrm{MHz}$ - and a narrow-band mode with a central frequency shifted to $4963 \mathrm{MHz}$ and a reduced bandwidth of $295 \mathrm{MHz}$. Raster scans with a speed of $4 \% \mathrm{~min}$ were made along the Galactic longitude (L) and latitude (B) directions. A single observation mostly lasted for about two hours, so that only a portion of the large target field was covered. Elevations were always high enough that ground radiation fluctuations can be largely avoided. Ten full coverages were processed and then calibrated by $3 \mathrm{C} 286$ and 3C 295, the main polarized and un-polarized calibrators. Finally, 

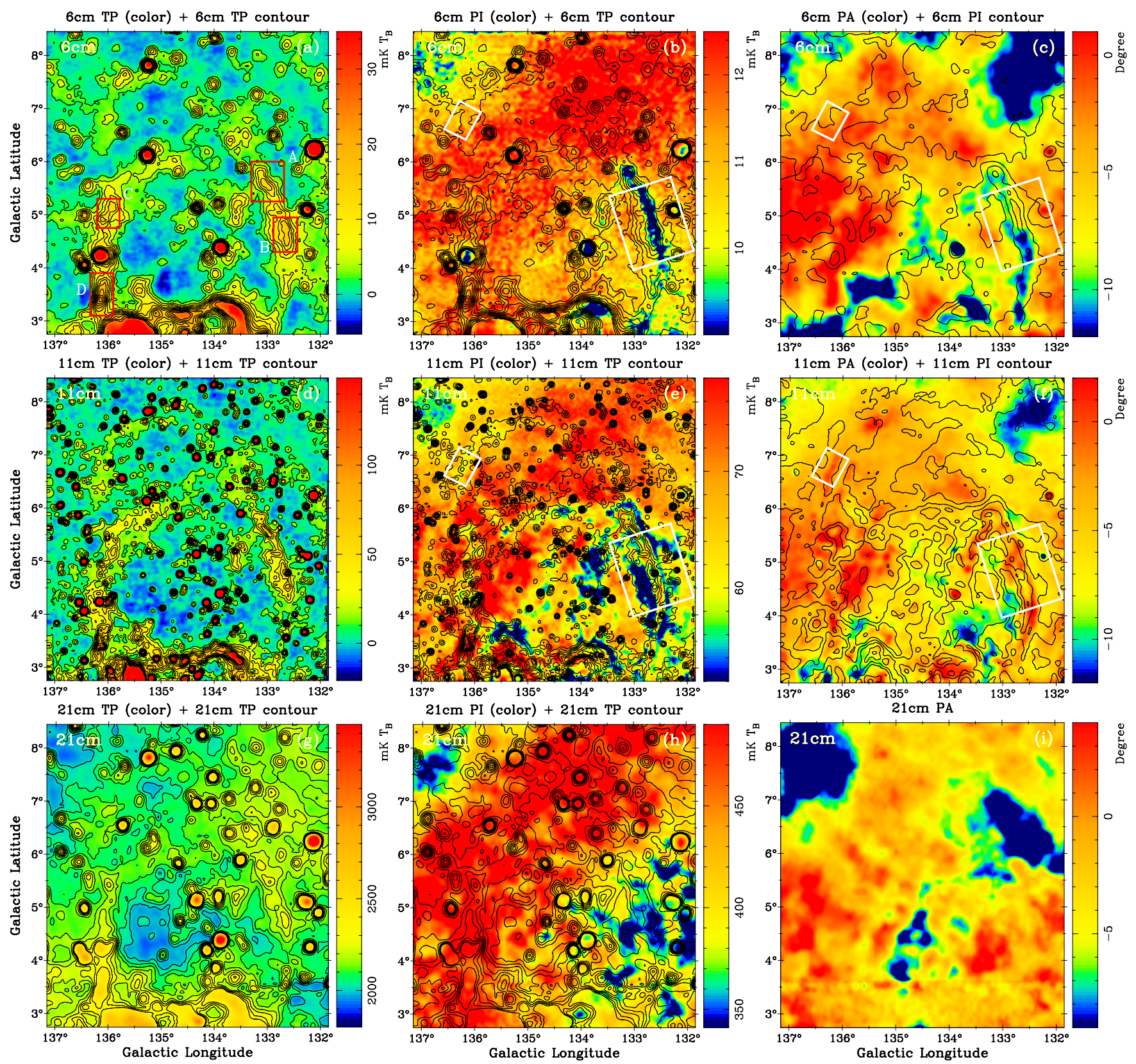

Fig. 1. Total intensity $I$, polarization intensity PI, and polarization angle PA images of the W4 superbubble at $\lambda 6 \mathrm{~cm}$ (upper panels a), b), c)), $\lambda 11 \mathrm{~cm}$ (middle panels $\mathbf{d}), \mathbf{e}), \mathbf{f})$ ), and $\lambda 21 \mathrm{~cm}$ (bottom panels $\mathbf{g}), \mathbf{h}), \mathbf{i})$ ). The angular resolutions are $9.5 \mathrm{for} \lambda 6 \mathrm{~cm}$ images in panels a) and b), 4.3 for $\lambda 11 \mathrm{~cm}$ images in panels d) and e), and 9.35 for $\lambda 21 \mathrm{~cm}$ images in panels $\mathbf{g}), \mathbf{h})$, and i). The PA images at $\lambda 6 \mathrm{~cm}($ panel $\mathbf{c}))$ and $\lambda 11 \mathrm{~cm}$ (panel f)) have angular resolutions of $12^{\prime}$ and $6^{\prime}$, respectively. The total intensity contours for $I$ and PI images run from $1.8 \mathrm{mK} T_{\mathrm{b}}$ in steps of $2.4 \mathrm{mK} T_{\mathrm{b}}$ for $\lambda 6 \mathrm{~cm}$, from $10.0 \mathrm{mK} T_{\mathrm{b}}$ in steps of $12.0 \mathrm{mK} T_{\mathrm{b}}$ for $\lambda 11 \mathrm{~cm}$ (after subtracting strong point-like sources), and from $2000 \mathrm{mK} T_{\mathrm{b}}$ in steps of $50 \mathrm{mK} T_{\mathrm{b}}$ for $\lambda 21 \mathrm{~cm}$. The PI contours on the PA images start from $5.0 \mathrm{mK} T_{\mathrm{b}}$ in steps of $1.0 \mathrm{mK} T_{\mathrm{b}}$ for $\lambda 6 \mathrm{~cm}$, and from $50.0 \mathrm{mK} T_{\mathrm{b}}$ in steps of $8.0 \mathrm{mK} T_{\mathrm{b}}$ for $\lambda 11 \mathrm{~cm}$. PI contours are not overlaid on the $\lambda 21 \mathrm{~cm}$ PA image, because no clear correlations can be found. The rectangles in panel a) are the regions for TT-plots study, while the rectangles in panels b), c), and e), f) indicate the regions used for the Faraday screen model fitting.

individual maps were combined following the procedures described by Gao et al. (2010). The final image of the W4 superbubble (see Fig. 1) is centred at $\ell=134.5, b=5^{\circ}$.6, covering an area of $5.3 \times 5^{\circ} .7$.

\subsection{Effelsberg $\lambda 11 \mathrm{~cm}$ data}

The $\lambda 11 \mathrm{~cm}$ observations were done in the summer of 2008 with the Effelsberg 100-m telescope. The receiving system was described by Uyanıker (2004), but was upgraded in 2005 with lower noise amplifiers and an eight-channel polarimeter. Here we made use of its broad-band channel with $80 \mathrm{MHz}$ bandwidth. Data processing and calibration follow the same procedures as for the $\lambda 6 \mathrm{~cm}$ observations.

\subsection{Combined $\lambda 21 \mathrm{~cm}$ data}

The $\lambda 21 \mathrm{~cm}$ data were extracted from the EMLS (Reich et al., in prep.) with a central frequency of $1400 \mathrm{MHz}$ and a bandwidth of $20 \mathrm{MHz}$. The missing large-scale structures were added by the Stockert $\lambda 21 \mathrm{~cm}$ survey data (Reich 1982; Reich \& Reich 1986) 
for total intensities, and the DRAO 26-m single-dish data (Wolleben et al. 2006) for polarization intensities.

We also added the available $\lambda 21 \mathrm{~cm}$ total intensity data from the Canadian Galactic plane survey (Taylor et al. 2003), the polarization data from Landecker et al. (2010), and the synthesis data from West et al. (2007), convolved to a 1.5 circular beam to the zero-level restored EMLS maps. A detailed description of the merging process of polarization data from the DRAO synthesis telescope, the Effelsberg 100-m and the DRAO 26-m telescopes was given by Landecker et al. (2010), whose procedure we followed. The resulting $\lambda 21 \mathrm{~cm}$ map does not fully cover the area of the $\lambda 6 \mathrm{~cm}, \lambda 11 \mathrm{~cm}$, and EMLS $\lambda 21 \mathrm{~cm}$ maps.

\subsection{Absolute zero-level restoration for the $\lambda 6 \mathrm{~cm}$ and $\lambda 11 \mathrm{~cm}$ polarization data}

Interferometric data miss short-spacings and single-dish maps are generally set on arbitrary zero levels. As emphasized by Reich (2006), missing large-scale structure means that diffuse polarized emission originating in the magnetized interstellar medium cannot be properly interpreted.

The observed polarization $U$ and $Q$ maps at $\lambda 6 \mathrm{~cm}$ and $\lambda 11 \mathrm{~cm}$ were set to zero at their boundaries and thus miss polarized emission from larger-scale components. The zerolevel problem was solved for the $\lambda 6 \mathrm{~cm}$ polarization data of the Urumqi survey (e.g. Sun et al. 2007; Gao et al. 2010) by adding the missing large-scale component by extrapolation of the WMAP $K$-band $(22.8 \mathrm{GHz})$ polarization data (Page et al. 2007; Hinshaw et al. 2009), which are on absolute levels. This is based on the assumption that Faraday rotation of the Galactic diffuse interstellar medium is negligible for frequencies as high as $4.8 \mathrm{GHz}$, which is valid except for the inner Galaxy (Sun et al. 2011). For the outer region within a few degrees away from the Galactic plane, such as the W4 superbubble, Faraday rotation effects can certainly be neglected between $\lambda 6 \mathrm{~cm}$ and the WMAP $K$-band.

For the W4 superbubble, we followed the same polarization zero-level restoration scheme as described by Gao et al. (2010), but using the most recent WMAP $K$-band 9 -yr data (Bennett et al. 2013). First, the Urumqi $\lambda 6 \mathrm{~cm}$ polarization $U$ and $Q$ maps and the corresponding WMAP $K$-band polarization maps were convolved to a common angular resolution of $2^{\circ}$. The convolved WMAP $U$ and $Q$ maps needed to be scaled to $\lambda 6 \mathrm{~cm}$ by a factor of $\left(\frac{4800}{22800}\right)^{\beta}$, where $\beta$ is the brightness temperature spectral index $\left(T_{\mathrm{b}} \sim \nu^{\beta}\right)$ for diffuse polarized emission. Spectral index maps for polarization intensities at $75^{\prime}$ resolution were derived from the combined $\lambda 21 \mathrm{~cm}$ (Effelsberg 100-m and DRAO 26-m) and the WMAP $K$-band data, and also for control for the WMAP $K$ - and $K a$-band $(33 \mathrm{GHz})$ data for the entire W4 superbubble area. In both cases, the mean of $\beta$ was close to -3 , i.e. for $\lambda 21 \mathrm{~cm}$ versus $K$-band, $\beta=-2.97 \pm 0.04$, and for $K$-band versus $K a$-band, $\beta=-2.98 \pm 0.31$, indicating that depolarization at $\lambda 21 \mathrm{~cm}$ is not important for large-scale emission. Finally, the $\beta$-scaled WMAP $U$ and $Q$ data at $2^{\circ}$ angular resolution were compared with the convolved $\lambda 6 \mathrm{~cm} U$ and $Q$ data, respectively. The differences were taken as the missing large-scale components and added to the observed $\lambda 6 \mathrm{~cm}$ data at their original angular resolution. The $\lambda 6 \mathrm{~cm}$ polarization intensity $(\mathrm{PI})$ was then calculated from the restored $U$ and $Q$ as $\mathrm{PI}=\sqrt{U^{2}+Q^{2}}$, and the polarization angle (PA) was obtained by $\mathrm{PA}=\frac{1}{2} \operatorname{atan}(U / Q)$.

The Faraday rotation effect at $\lambda 11 \mathrm{~cm}$ is about 3.4 times that of $\lambda 6 \mathrm{~cm}$ ( $\lambda^{2}$ dependence), so a successful zero-level

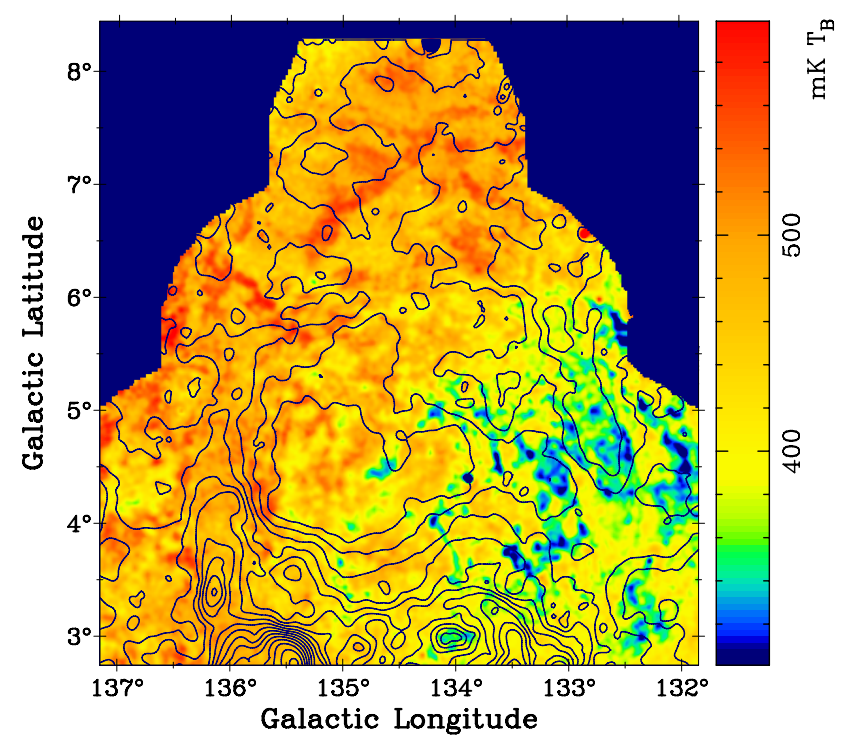

Fig. 2. Combined $\lambda 21 \mathrm{~cm}$ polarized emission of the W4 superbubble with data from the DRAO interferometer, the Effelsberg 100-m, and the DRAO 26-m single-dish telescopes at an angular resolution of $3^{\prime}$. Overlaid $\lambda 21 \mathrm{~cm}$ contours are from the source subtracted and spatially filtered total intensity map (see text). Contour lines run from $2000 \mathrm{mK}$ $T_{\mathrm{b}}$ in steps of $50 \mathrm{mK} T_{\mathrm{b}}$.

recovery using high-frequency WMAP $K$-band data requires a careful check on the rotation measure $(\mathrm{RM})$ in the area of interest. For the W4 superbubble area, we calculated a RM map between $\lambda 21 \mathrm{~cm}$ (Effelsberg + DRAO) and $\lambda 1.3 \mathrm{~cm}$ (WMAP $K$-band) from the polarization angle data: $\mathrm{RM}=$ $\left(\mathrm{PA}_{1.3 \mathrm{~cm}}-\mathrm{PA}_{21 \mathrm{~cm}}\right) /\left(0.013^{2}-0.214^{2}\right)$ at an angular resolution of $75^{\prime}$. The RMs are found to be small in general and the mean is $0.74 \pm 1.01 \mathrm{rad} \mathrm{m}^{-2}$, which causes only small deviations in PA of $-1^{\circ}$ to 3.3 between the Effelsberg $\lambda 11 \mathrm{~cm}$ and the WMAP $\lambda 1.3 \mathrm{~cm}$ data. Therefore, we restored the missing large-scale polarized emission for the Effelsberg $\lambda 11 \mathrm{~cm}$ data by the same procedures as for the Urumqi $\lambda 6 \mathrm{~cm}$ data.

\section{Results}

We present the total intensity $I$, the polarization intensity PI of the W4 superbubble at $\lambda 6 \mathrm{~cm}, \lambda 11 \mathrm{~cm}$, and $\lambda 21 \mathrm{~cm}$ at angular resolutions of $9.5,4.3$, and 9.35 in Fig. 1, where the $\lambda 21 \mathrm{~cm}$ images are from the combined EMLS and DRAO 26-m single dish map. The angular resolutions of the $\lambda 6 \mathrm{~cm}$ and $\lambda 11 \mathrm{~cm}$ PA images are convolved to $12^{\prime}$ and $6^{\prime}$, respectively, to increase the signal-to-noise ratio. The $\lambda 21 \mathrm{~cm}$ PA image is shown at its original resolution of 9:35. A higher angular resolution $\lambda 21 \mathrm{~cm}$ PI map combined from the DRAO synthesis telescope (West et al. 2007), the EMLS, and the DRAO 26-m telescope convolved to a $3^{\prime}$ circular beam is displayed in Fig. 2. This map is overlaid with contours of a source-subtracted and unsharp-masked $\lambda 21 \mathrm{~cm}$ total intensity map, which was combined from DRAO synthesis telescope, EMLS, and Stockert survey data. The singled-out PI images for the western ${ }^{1}$ shell of the W4 superbubble at $\lambda 6 \mathrm{~cm}$, $\lambda 11 \mathrm{~cm}$, and $\lambda 21 \mathrm{~cm}$ are shown in Fig. 3. For all the images, the $\lambda 6 \mathrm{~cm}$ and $\lambda 11 \mathrm{~cm}$ total intensity images are the observed

\footnotetext{
The rotation angle between the Galactic plane and the Celestial equator is small $\left(\sim 25^{\circ}\right)$ in the W4 superbubble area, we thus refer to the larger longitudes as "east", and the smaller longitudes as "west" in this work.
} 

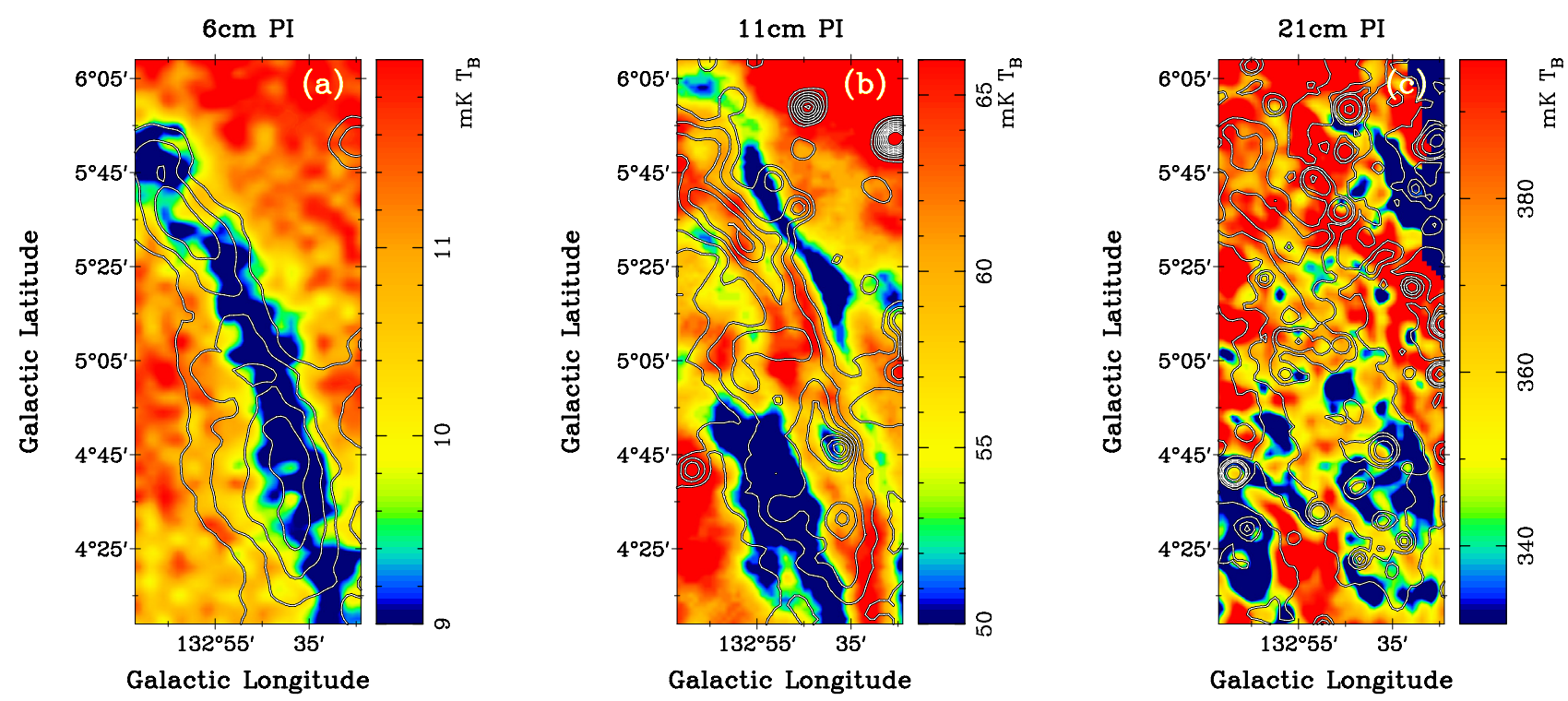

Fig. 3. Depolarization along the western shell of the $\mathrm{W} 4$ superbubble at $\lambda 6 \mathrm{~cm}$ (panel a)), where total intensity contours start at $4.9 \mathrm{mK} T_{\mathrm{b}}$ and run in steps of $3.0 \mathrm{mK} T_{\mathrm{b}}$, and at $\lambda 11 \mathrm{~cm}$ (panel b)) where total intensity contours start from $9.0 \mathrm{mK} T_{\mathrm{b}}$ in steps of $13.5 \mathrm{mK} T_{\mathrm{b}}$, and at $\lambda 21 \mathrm{~cm}$ (panel c)) where the total intensity (DRAO interferometer + Effelsberg + DRAO 26-m) contours run from $2000+(n-1) \times 50 \mathrm{mK} T_{\mathrm{b}}(n=1,2$, $3,4)$, and $2500+(n-5) \times 800 \mathrm{mK} T_{\mathrm{b}}(n=5,6,7,8)$. The angular resolutions for the $\lambda 6 \mathrm{~cm}, \lambda 11 \mathrm{~cm}$, and $\lambda 21 \mathrm{~cm}$ images are 9.5, 4.3, and $3^{\prime}$, respectively.

ones on relative zero levels, while all the others are restored to absolute zero levels.

\subsection{Total intensity emission and spectral information}

In all total intensity images, the W4 superbubble shows up by two bright ridges emerging from the H II region complex W4 at about $\ell \sim 133^{\circ}$ and $\ell \sim 136^{\circ}$, extending towards high latitudes. These two ridges form a loop structure, since they close at about $b=6^{\circ}$. From the $\lambda 6 \mathrm{~cm}$ and $\lambda 11 \mathrm{~cm}$ images, a faint highlatitude extension of the western shell can be traced to more than $b=7^{\circ}$. Another partial shell-like structure can be distinguished above the major eastern shell of the W4 superbubble and merges with the western extension at about $b=8^{\circ}$. According to the apparent size determined by the maximum lengths on the Galactic longitude and latitude dimensions, $\Delta \ell \times \Delta b=3.8 \times 7.1(\Delta b$ is calculated with respect to the position of $\mathrm{OCl} 352$ at $b=0.9)$, the physical size of the W4 superbubble is about $150 \mathrm{pc}$ in width and $270 \mathrm{pc}$ in height for a distance of $2.2 \mathrm{kpc}$. These results are consistent with previous estimates (e.g. Dennison et al. 1997).

Based on the tight correlation between radio continuum and $\mathrm{H} \alpha$ emission and the flat radio spectrum derived between $408 \mathrm{MHz}$ and $1420 \mathrm{MHz}$, West et al. (2007) conclude that the radio continuum emission from the shells of the W4 superbubble is optically thin thermal emission. We confirmed this by deriving the spectral index from the present data using TT-plots (Turtle et al. 1962) between $1.4 \mathrm{GHz}$ and $4.8 \mathrm{GHz}$. After subtracting the NVSS sources (Condon et al. 1998), the brightness temperature spectral index between $\lambda 6 \mathrm{~cm}$ and $\lambda 11 \mathrm{~cm}$ was found to be $\beta=-1.98 \pm 0.16$ for the western shell and $\beta=-2.04 \pm 0.26$ for the eastern shell (Fig. 4, left panels). Between $\lambda 6 \mathrm{~cm}$ and $\lambda 21 \mathrm{~cm}$, we obtained $\beta=-2.12 \pm 0.39$ for region " $\mathrm{A}$ ", $\beta=-2.19 \pm 0.38$ for region " $\mathrm{B}$ ", and $\beta=-2.16 \pm 0.33$ and $\beta=-2.16 \pm 0.10$ for the regions " $C$ " and " $D$ ", respectively for the marked regions in Fig. 1. These results agree with optical-thin thermal emission, which we use for the passive Faraday screen model to derive the magnetic fields within the shells of the W4 superbubble in Sect. 4.2.

\subsection{Polarization}

Unlike the resemblance in the total intensity images shown for the three different wavelengths, polarization behaves much differently. At $\lambda 6 \mathrm{~cm}$, the most pronounced feature related to the W4 superbubble is the strong depolarization that nicely follows the western shell and the PA deviation across the shell. No other prominent and closely related PI and PA features are seen along any other shell structures.

The $\lambda 11 \mathrm{~cm}$ polarization image discloses more structural details owing to its higher angular resolution. The polarization intensities and angles vary strongly in the western shell area of the W4 superbubble (see Fig. 3). Other than at $\lambda 6 \mathrm{~cm}$, depolarization is also seen for some sections of the eastern shell and the high-latitude filament centred approximately at $\ell=136^{\circ}, b=7^{\circ}$. This, in conjunction with the $\lambda 6 \mathrm{~cm}$ PI image, implies that RM varies in values and regularity over the W4 superbubble and is smaller in the eastern shell than in the western shell. Polarization structures are also visible in the bubble's interior. A notable feature is the arc-like structure running from longitude $133^{\circ}$ to $134^{\circ}$ and latitude 6.0 to 4.5 , which is also visible but fainter at $\lambda 6 \mathrm{~cm}$.

At $\lambda 21 \mathrm{~cm}$, no strong structural coincidence can be identified between total intensity and polarization at first glance. West et al. (2007) saw the western and eastern shells of the W4 superbubble in depolarization in their $\lambda 21 \mathrm{~cm}$ map using DRAO synthesis data alone. When averaging their data for a $10^{\prime}$ wide latitude stripe centred at $b=4.5$, the polarization intensity for the eastern shell drops from a surrounding level of about $65 \mathrm{mK}$ $T_{\mathrm{b}}$ to $40 \mathrm{mK} T_{\mathrm{b}}$, and for the western shell it decreases from $50 \mathrm{mK} T_{\mathrm{b}}$ to about $40 \mathrm{mK} T_{\mathrm{b}}$. When combining the DRAO synthesis telescope data with the Effelsberg and DRAO singledish data (Fig. 2), we found that this depolarization is much less pronounced than the pure DRAO synthesis telescope data. The 

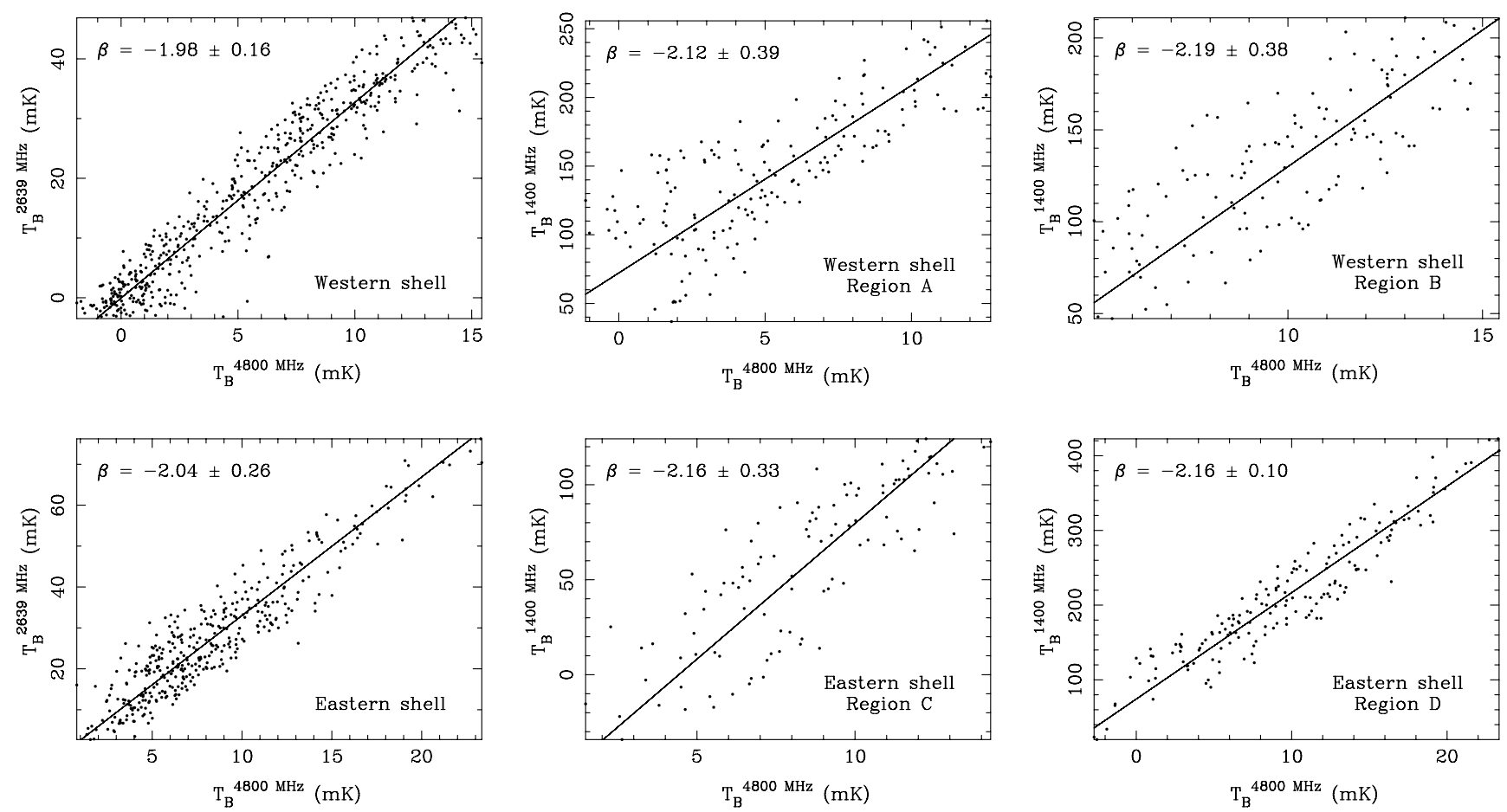

Fig. 4. TT-plot results for the western shell (upper panels) and the eastern shell (lower panels) of the W4 superbubble between $\lambda 6 \mathrm{~cm}$ and $\lambda 11 \mathrm{~cm}$ (left panels) and for the four regions (A, B, C, and D) in the shells (see Fig. 1) between $\lambda 6 \mathrm{~cm}$ and $\lambda 21 \mathrm{~cm}$.

reason is the much higher absolute polarization level of about $500 \mathrm{mK} T_{\mathrm{b}}$ for the eastern and $350 \mathrm{mK} T_{\mathrm{b}}$ for the western shell. Fluctuations of the Galactic polarized emission seem to mask the depolarization along the W4 superbubble shells. For a zoomed view of the western shell at $3^{\prime}$ resolution (Fig. 3), there is, however, a morphological coincidence between total and polarization intensity, indicating that related depolarization exists.

On larger scales, an inclined broad stripe of strong polarized emission runs across the image from the lower left (southeast) to the upper right (north-west) corner, while depolarization patches are seen in the orthogonal areas of the image. Both the Effelsberg and the DRAO single-dish data independently show this feature at $\lambda 21 \mathrm{~cm}$. This general distribution is also seen in the $\lambda 6 \mathrm{~cm}$ and $\lambda 11 \mathrm{~cm}$ polarization data that are corrected by large-scale emission based on the WMAP $K$-band data (see Fig. 1).

\section{The magnetic field strength in the W4 superbubble shell}

Regular changes in sign or values of RMs of extragalactic sources across a finite area often indicate the existence of a Galactic Faraday screen. Sufficient RM sources in a sky area can be used to determine the line-of-sight component of the magnetic fields within such foreground screens (e.g. Harvey-Smith et al. 2011). There are 40 sources with determined RMs within the W4 superbubble area in the catalogue of Xu \& Han $(2014)^{2}$ supplemented by the NVSS RM catalogue (Taylor et al. 2009). Most of these RMs show negative signs, resulting from the orientation of the Galactic magnetic field running clockwise (viewed from the north Galactic pole) in this part of the Perseus arm (Han et al. 2006). Five RMs are positive, which might indicate

\footnotetext{
2 http://zmtt.bao.ac.cn/RM/index.html
}

small-scale variations or source-intrinsic properties. However, the present RM number density is too sparse to estimate the magnetic field for shell regions of the W4 superbubble.

\subsection{The Faraday screen model}

A passive Faraday screen is a magneto-ionized bubble, filament, or sheet that does not emit polarized emission, but modulates background polarized emission passing through it via the Faraday effect. A successful model for deriving the physical parameters of these Faraday screens was developed by Sun et al. (2007) for screens detected in the Urumqi $\lambda 6 \mathrm{~cm}$ survey. It was also applied in several follow-up studies (e.g. Gao et al. 2010; Xiao et al. 2011). In its simplest form, the model takes the observational fact that the foreground and background Galactic magnetic field of the screens have the same orientation and PA $\sim 0^{\circ}$, i.e. parallel to the Galactic plane at high enough frequencies. We have verified in Sect. 3.1 that the W4 superbubble exclusively emits optically thin thermal emission. We now check whether foreground and background PAs around the W4 superbubble area meet the condition of PA $\sim 0^{\circ}$. Starlight polarization observations of the W4 superbubble area (Heiles 2000) support the idea that the Galactic magnetic fields are aligned to the Galactic plane $\left(\mathrm{PA}=-1^{\circ} \pm 7^{\circ}\right)$ for a distance range from $30 \mathrm{pc}$ to $2.85 \mathrm{kpc}$. This is further supported by the low-frequency polarization measurements for the large excessive "Fan" region (Spoelstra 1984), covering the W4 superbubble area, and also by the WMAP $K$ - and $K a$-band polarization data, where Galactic RMs in this part of the Galaxy are small, and the large-scale Galactic magnetic fields (Han et al. 2006) will only marginally change PAs from their intrinsic values. In addition, we also carried out $\lambda 6 \mathrm{~cm}$ simulations in the direction of the W4 superbubble using the Sun et al. (2008) and Sun \& Reich (2010) Galactic 3D-emission model, which is based on all-sky surveys, 


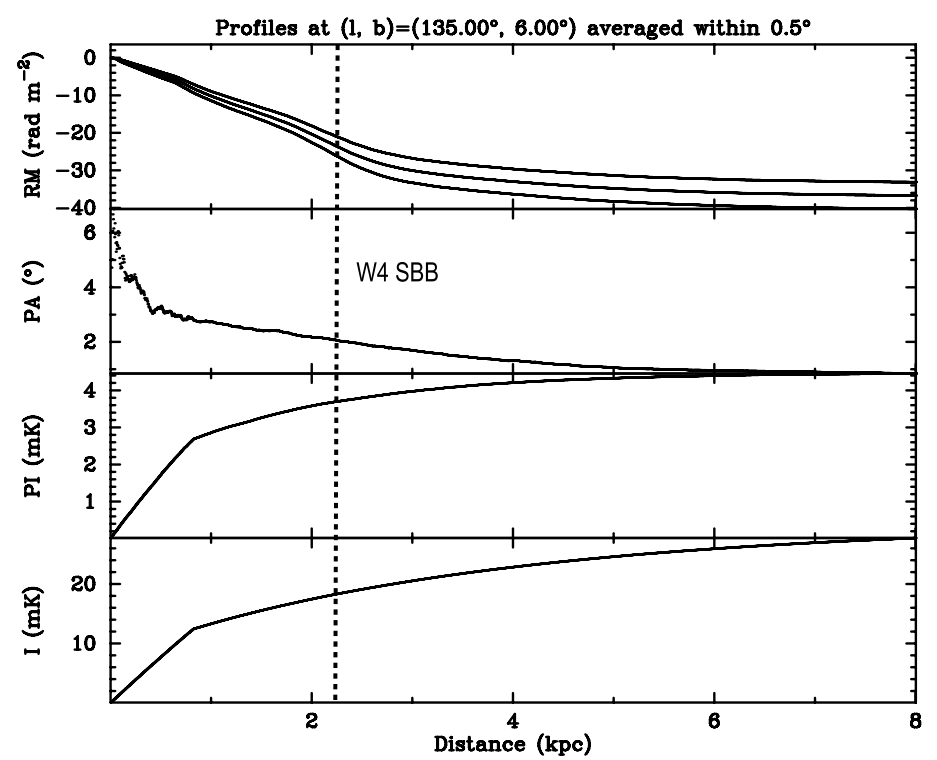

Fig. 5. Simulations in the direction $\ell=135^{\circ}, b=6^{\circ}$ showing accumulated and spatial averaged RM (including one $\sigma$ errors), PA, PI, and $I$ values at $\lambda 6 \mathrm{~cm}$ as a function of distance from the Sun.

RMs, and thermal emission data and also includes realistic magnetic field turbulence (Sun \& Reich 2009). The simulation result is shown in Fig. 5. Foreground PA of the W4 superbubble at $2.2 \mathrm{kpc}$ is close to zero and differs by just about $1^{\circ}$ from the PA of the background emission at distances of $8 \mathrm{kpc}$ or more. We repeated the simulation for various directions within the W4 superbubble area at $\lambda 6 \mathrm{~cm}$, and obtained very similar results. Simulations at $\lambda 11 \mathrm{~cm}$ and $\lambda 21 \mathrm{~cm}$ show very small PA differences as well. We concluded that the Galactic 3D-emission simulations also support that the assumptions required for the passive Faraday screen model are satisfied.

The passive Faraday screen model uses three parameters: a depolarization factor $f$, which describes the reduction of the background polarization by the Faraday screen; the Faraday screen foreground polarized emission fraction $c$ with respect to the observed polarized emission at an off-position away from the screen; and the polarization rotation angle within the screen $\psi_{\mathrm{s}}$, which is the parameter for calculating $\mathrm{RM}(\mathrm{RM}=$ $\psi_{\mathrm{s}} / \lambda^{2}$ ), and the line-of-sight component of the magnetic field (see Eq. (6)). These three parameters fit two observables: (1) the ratio $\mathrm{PI}_{\text {on }} / \mathrm{PI}_{\text {off }}$ and (2) the angle difference $\mathrm{PA}_{\text {on }}-\mathrm{PA}_{\text {off }}$, where "on" and "off" denote that the line of sight passes through (on) or not (off) the screen. The Faraday screen model equation is given in Eq. (1) (see Sun et al. 2007, for a detailed derivation):

$$
\left\{\begin{array}{l}
\frac{\mathrm{PI}_{\mathrm{on}}}{\mathrm{PI}_{\mathrm{off}}}=\sqrt{f^{2}(1-c)^{2}+c^{2}+2 f c(1-c) \cos 2 \psi_{\mathrm{s}}}, \\
\mathrm{PA}_{\mathrm{on}}-\mathrm{PA}_{\mathrm{off}}=\frac{1}{2} \arctan \left(\frac{f(1-c) \sin 2 \psi_{\mathrm{s}}}{c+f(1-c) \cos 2 \psi_{\mathrm{s}}}\right) .
\end{array}\right.
$$

In the following we allow $f$ to be in the range $[0.00,1.00]$ and $c$ between 0.6 to 1.0 , when taking the simulation result (Fig. 5) into account. These two parameters vary in steps of $0.01 . \psi_{\mathrm{s}}$ is searched for in the range $\left[-90^{\circ}, 90^{\circ}\right]$ in steps of $1^{\circ}$.

\subsection{Analysis of the western shell}

The clearest Faraday effect for the W4 superbubble is the depolarized western shell seen at $\lambda 6 \mathrm{~cm}$ and $\lambda 11 \mathrm{~cm}$. With the measurements at these two wavelengths, we estimated the
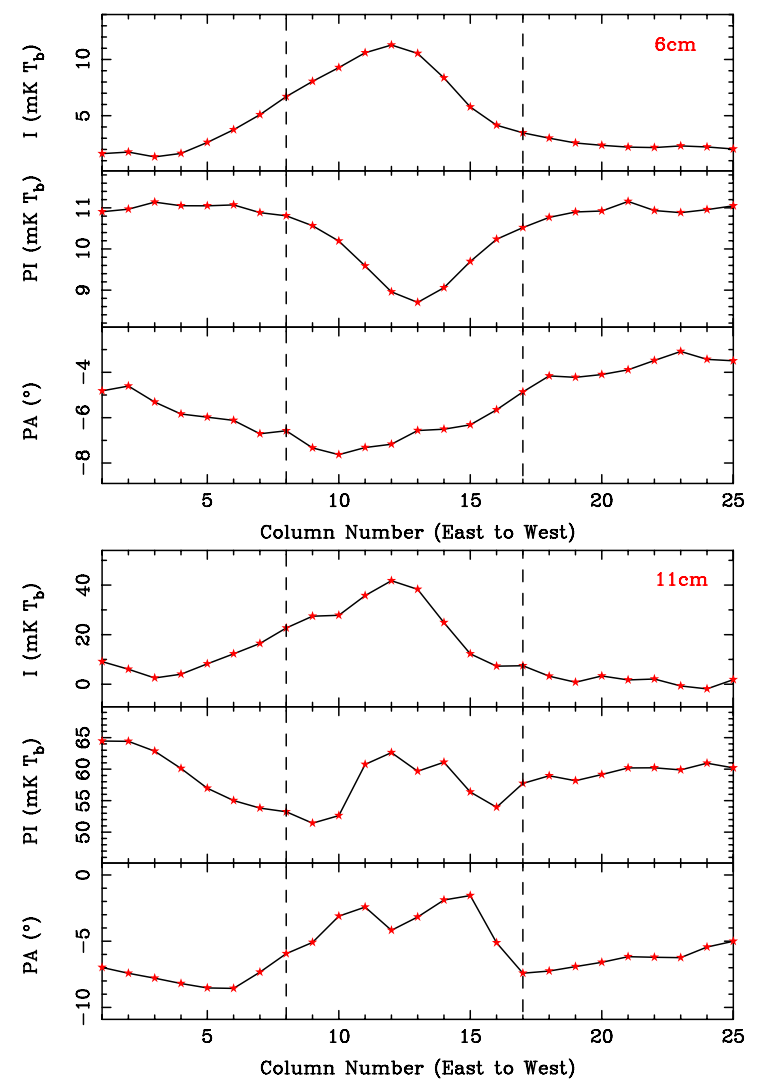

Fig. 6. Upper panel: profiles of the $\lambda 6 \mathrm{~cm} \mathrm{I} \mathrm{(relative} \mathrm{zero} \mathrm{level),}$ PI (absolute zero level), and PA (absolute zero level) averaged perpendicular to the western shell of the W4 superbubble within the area marked by the large white rectangular region in Fig. 1. The pixel size is $3^{\prime}$. The vertical dashed lines indicate the "on - off" boundaries of the Faraday screen. Lower panel: the same as the upper panel, but for the Effelsberg $\lambda 11 \mathrm{~cm}$ data.

line-of-sight component of the magnetic field $B_{/ /}$based on the Faraday screen model described above for a section as indicated by the large white rectangle in Fig. 1. Averaged $\lambda 6 \mathrm{~cm}$ and $\lambda 11 \mathrm{~cm} \mathrm{I,PI,} \mathrm{and} \mathrm{PA} \mathrm{profiles} \mathrm{perpendicular} \mathrm{to} \mathrm{the} \mathrm{western}$ shell were calculated and shown in Fig. 6. The shell direction is inclined, and the averaged stripes or columns run parallel to it. We labelled the result by the column number from east to west across the western shell (see Fig. 6). The typical character of a passive Faraday screen is seen at $\lambda 6 \mathrm{~cm}$; i.e., an apparent PI depression is identified with its minimum close to the peak position of the total intensity $I$. The very small PA difference between "on" and "off" positions implies a large foreground fraction $c$ of the polarized emission.

To calculate the two observables, $\mathrm{PI}_{\text {on }} / \mathrm{PI}_{\text {off }}$ and $\mathrm{PA}_{\text {on }}-\mathrm{PA}_{\text {off }}$, the "on" and "off" positions of the Faraday screen must be determined first. Combining the profiles of the $\lambda 6 \mathrm{~cm}$ and $\lambda 11 \mathrm{~cm}$ data, we set the boundary of "on" and "off" positions of the Faraday screen as marked in Fig. 6. The column size of 3' corresponds to $1.9 \mathrm{pc}$ for a distance of $2.2 \mathrm{kpc}$ of the W4 superbubble. The $\lambda 6 \mathrm{~cm} \mathrm{PI}_{\text {off }}$ and $\mathrm{PA}_{\text {off }}$ were calculated as the average values of PIs and PAs on the "off" positions (Cols. 1-8 and 17-25, see Fig. 6 upper panel). The central eight (9th to 16th, Fig. 6 and Table 2) columns represent the interior of the Faraday screen and were used for model fitting.

We assumed $1 \sigma$ observational uncertainty for the $\lambda 6 \mathrm{~cm}$ observations and used Eq. (1) for the Faraday screen model fitting. A series of $\left(f, c, \psi_{\mathrm{s}}\right)$ results were obtained, which are consistent 
A\&A 578, A24 (2015)

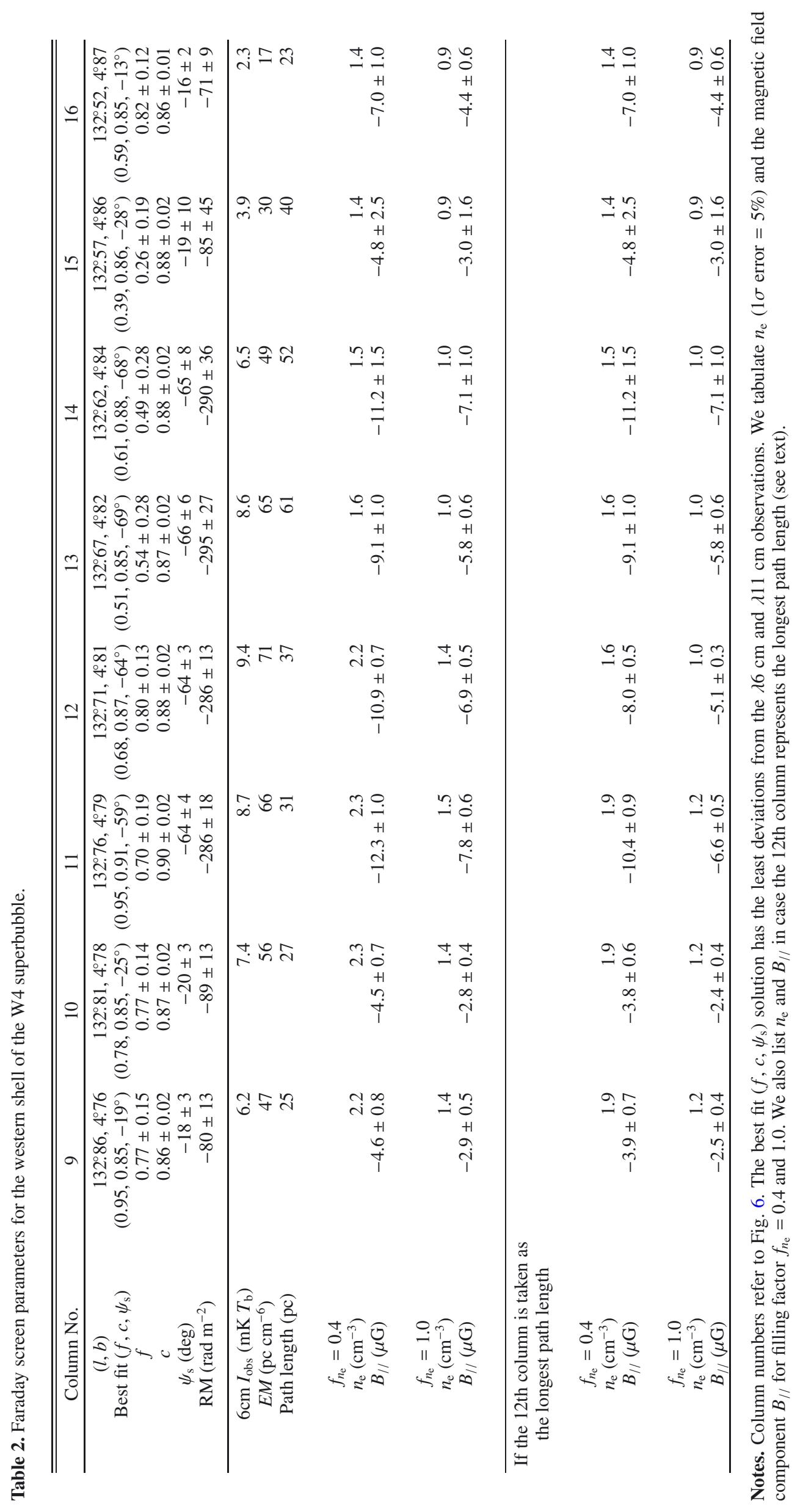


with the $\lambda 6 \mathrm{~cm}$ observables $\mathrm{PI}_{\text {on }} / \mathrm{PI}_{\text {off }}$ and $\mathrm{PA}_{\text {on }}-\mathrm{PA}_{\text {off }}$. We then used these qualified $\left(f, c, \psi_{\mathrm{s}}\right)$ to predict $\lambda 11 \mathrm{~cm} \mathrm{PI}$ $\left(\mathrm{PI}=\sqrt{U^{2}+Q^{2}}\right)$ and $\mathrm{PA}_{\text {on }}\left(\mathrm{PA}=\frac{1}{2} \operatorname{atan} \frac{U}{Q}\right)$ based on the following equation (for details see Sun et al. 2007):

$$
\left\{\begin{array}{l}
U_{\mathrm{on}}=f \mathrm{PI}_{\mathrm{bg}} \sin 2 \psi_{\mathrm{s}}, \\
Q_{\mathrm{on}}=\mathrm{PI}_{\mathrm{fg}}+f \mathrm{PI}_{\mathrm{bg}} \cos 2 \psi_{\mathrm{s}} .
\end{array}\right.
$$

Here, $\mathrm{PI}_{\mathrm{fg}, 11 \mathrm{~cm}}=\mathrm{PI}_{\text {off }, 11 \mathrm{~cm}} \times c$ and $\mathrm{PI}_{\mathrm{bg}, 11 \mathrm{~cm}}=\mathrm{PI}_{\text {off }, 11 \mathrm{~cm}} \times$ $(1-c)$, where $\lambda 11 \mathrm{~cm} \mathrm{PI}{ }_{\text {off, } 11 \mathrm{~cm}}$ was calculated as $\mathrm{PI}_{\mathrm{off}, 11 \mathrm{~cm}}=$ $\mathrm{PI}_{\text {off }, 6 \mathrm{~cm}}\left(\frac{2639 \mathrm{MHz}}{4800 \mathrm{MHz}}\right)^{\beta}=65.8 \mathrm{mK} T_{\mathrm{b}}$, with a spectral index $\beta=-3$ as obtained in Sect. 2.4. This agrees with the measured values at the edges of the $\lambda 11 \mathrm{~cm}$ PI profile in Fig. 6. The rotation angles at $\lambda 11 \mathrm{~cm}$ were calculated as $\psi_{11 \mathrm{~cm}}=\psi_{6} \mathrm{~cm}\left(\frac{0.114}{0.0625}\right)^{2}$. We compared the predicted values with the $\lambda 11 \mathrm{~cm}$ observational results within $1 \sigma$ errors, which further filtered the $\left(f, c, \psi_{\mathrm{s}}\right)$ data to agree with both $\lambda 6 \mathrm{~cm}$ and $\lambda 11 \mathrm{~cm}$ observations. From all the remaining results, we found the parameter $c$ to be rather well constrained within 0.8 to 0.9 (see Table 2 ) and largely independent of $f$ variations. This is consistent with the dominant foreground contribution towards the W4 superbubble. The reduction of the polarized background emission is mathematically possible in various ways. Here, $\psi_{\mathrm{s}}$ is the most interesting parameter and is also well constrained, which enables us to make a reasonable estimate of the magnetic field and assess its errors. In Table 2, we listed the average values of $f, c$, and $\psi_{\mathrm{s}}$ for each column in the Faraday screen, including standard deviation. We searched for the best fit $\left(f, c, \psi_{\mathrm{s}}\right)$ for each column by the least $\chi^{2}$ method. The difference between the average and the best fit value $(f$, $\left.c, \psi_{\mathrm{s}}\right)$ is small, with the maximum of $1.9 \sigma$. In the following, we used the averaged $\psi_{\mathrm{s}}$ and its standard deviation to derive the magnetic field within the western shell of the W4 superbubble.

The $\lambda 21 \mathrm{~cm}$ observations cannot be used for the model fitting since depolarization occurs at $\lambda 21 \mathrm{~cm}$ even by smaller RMs within the shell. However, it can be examined to verify the fit result. Considering the small-scale RM structures as shown by the $\lambda 11 \mathrm{~cm}$ data, total depolarization could be expected for the background PI at $\lambda 21 \mathrm{~cm}$ passing through the bubble's shell. In fact, the interferometric $\lambda 21 \mathrm{~cm}$ image by West et al. (2007) shows the depolarization along the W4 superbubble shells, which is almost masked in the single-dish polarization image as discussed in Sect. 3.2. In the West et al. (2007) interferometric map, the depolarization averaged for a $10^{\prime}$ wide latitude stripe is about $25 \mathrm{mK} T_{\mathrm{b}}$ along the eastern and about $10 \mathrm{mK} T_{\mathrm{b}}$ along the western shell. The single-dish map polarization level of about $500 \mathrm{mK} T_{\mathrm{b}}$ in the eastern and about $350 \mathrm{mK} T_{\mathrm{b}}$ in the western shell area imply an upper limit of about 0.97 for $c$ in the western shell and about 0.95 in the eastern shell. The large difference in the polarization emission levels in the interferometric and in the single-dish maps means that a quantitative analysis of the interferometric polarization data without large-scale correction cannot be made to estimate the field strength. We concluded that the $\lambda 21 \mathrm{~cm}$ polarization data agree with the parameters of the Faraday screen model along the western shell based on the $\lambda 6 \mathrm{~cm}$ and $\lambda 11 \mathrm{~cm}$ observations.

For an estimate of $B_{/ /}$within the western shell, the thermal electron density $n_{\mathrm{e}}$, its filling factor $f_{n_{\mathrm{e}}}$, and the path length $l$ within the bubble's shell are needed. Emission measure $(E M)$, which is the integral of $f_{n_{\mathrm{e}}}$ and $n_{\mathrm{e}}^{2}$ over the path length $\mathrm{d} l$ can be calculated, because it is related to optically-thin thermal radio continuum emission by the following equation (Wilson et al. 2013):

$$
\left(\frac{T_{\mathrm{b}}}{\mathrm{K}}\right)=8.235 \times 10^{-2}\left(\frac{T_{\mathrm{e}}}{\mathrm{K}}\right)^{-0.35}\left(\frac{v}{\mathrm{GHz}}\right)^{-2.1}\left(\frac{E M}{\mathrm{pc} \mathrm{cm}^{-6}}\right) a(v, T),
$$

where, $T_{\mathrm{b}}$ is the radio continuum brightness temperature measured at frequency $v, a(v, \mathrm{~T})$ is a factor close to 1 . We assumed $T_{\mathrm{e}}=8000 \mathrm{~K}$ for the ionized Galactic interstellar medium in general. Based on the $\lambda 6 \mathrm{~cm}$ observation, we deduced the $E M \mathrm{~s}$ within the western shell of the W4 superbubble. However, from Fig. 6 we saw a large-scale emission component of about $1.9 \mathrm{mK} T_{\mathrm{b}}$ at $\lambda 6 \mathrm{~cm}$ besides thermal emission $I$ from the western shell. After subtracting this large-scale component, EMs of the central eight columns within the screen area were calculated based on Eq. (3), as listed in Table 2.

To verify the $E M$ results that we obtained from the radio continuum data, $\mathrm{H} \alpha$ measurements were used to estimate the $E M \mathrm{~s}$ independently following Haffner et al. (1998) and Finkbeiner (2003) by using

$E M=2.75 T_{4}^{0.9} I_{\mathrm{H} \alpha} \exp [2.44 E(B-V)]$.

Dennison et al. (1997) measured $\mathrm{H} \alpha$ emission for several positions towards the W4 superbubble. At their position $K(\ell \sim$ $\left.133^{\circ} 0, b \sim 5.4\right)$, they observed an intensity of 5.8 Rayleigh. Reynolds et al. (2001) measured $6.9 \pm 0.5$ Rayleigh at the position $\ell=132^{\circ} .8, b=5$. 1 . Both directions are within the western shell region of the W4 superbubble. The extinction parameter $E(B-V)$, which must be taken into account, is in general difficult to obtain and often ignored for regions outside the Galactic plane. Two stars, Hilt $266\left(\ell=132^{\circ} .2, b=3^{\circ} .1\right)$ and Hilt 338 $\left(\ell=134^{\circ} .2, b=3^{\circ} .0\right)$, were found near the W4 superbubble with distances of $2.5 \mathrm{kpc}$ and $2.3 \mathrm{kpc}$, respectively. They have a measured extinction value of $E(B-V)=0.66$ (Hiltner 1956). Based on Eq. (4) and assuming $T=8000 \mathrm{~K}$, we derived the $E M \mathrm{~s}$ for the two positions as $65 \mathrm{pc} \mathrm{cm}^{-6}$ and $77.7 \pm 5.6 \mathrm{pc} \mathrm{cm}^{-6}$, which are consistent with those based on radio continuum data in Table 2.

The path lengths for the central eight columns (9th-16th) across the western shell of the W4 superbubble are related to the geometry, where we assume a uniform shell. The largest RM corresponds to the longest path length (Vallée 1982), which is corresponding to the 13th column in our case (see Fig. 6 and Table 2), and defines the inner radius of the shell. We also considered the case of a thermal shell, where the strongest total intensity emission is at the 12 th column, and included the results in Table 2. The angle between the central axis of the W4 superbubble and the 13th column is about 1.7 , and the angle between the 13th column and the outer edge of the western shell is 10.5. Then for a distance of $2.2 \mathrm{kpc}$, we calculated the inner and outer radii of the shell as $65 \mathrm{pc}$ and $72 \mathrm{pc}$, respectively. With a column size of $3^{\prime}$, the path lengths of the central eight columns were deduced. The electron densities and the strength of the line-of-sight component of the magnetic field $B_{/ /}$can be obtained following the equations given by Harvey-Smith et al. (2011), where the filling factor $f_{n_{\mathrm{e}}}$ is the only unknown parameter:

$$
\begin{aligned}
n_{\mathrm{e}} & =\sqrt{\frac{E M}{f_{n_{\mathrm{e}}} l}} \mathrm{~cm}^{-3}, \\
B_{/ /} & =\frac{R M}{0.81 \sqrt{E M} \sqrt{f_{n_{\mathrm{e}}}}} \mu \mathrm{G} .
\end{aligned}
$$

The filling factor is often assumed to be 1.0, as in West et al. (2007), while Harvey-Smith et al. (2011) used $f_{n_{\mathrm{e}}}=0.1$ for the sample of H II regions they studied. Since no well-determined value has been reported for superbubbles so far, we calculated $n_{\mathrm{e}}$ and $B_{/ /}$based on Eqs. (5) and (6), for $f_{n_{\mathrm{e}}}=0.1,0.4,0.7$, and 1.0. We used the standard error propagation to estimate the errors in $n_{\mathrm{e}}$ and $B_{/ /}$. The uncertainty of $n_{\mathrm{e}}$ results from the error of 

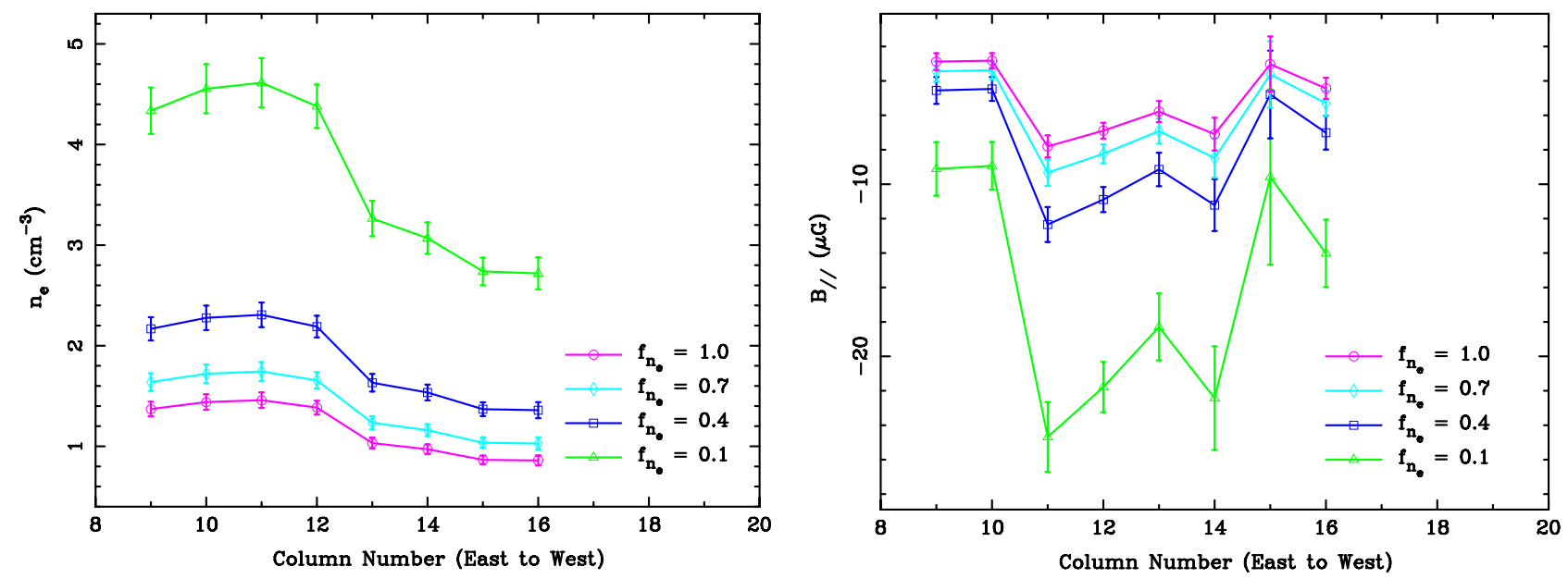

Fig. 7. Left panel: electron density $n_{\mathrm{e}}$ across the western shell of the W4 superbubble for filling factors $f_{n_{\mathrm{e}}}=0.1$ (green triangle), 0.4 (blue square), 0.7 (cyan diamond), and 1.0 (magenta circle). Right panel: the strength of the line-of-sight component of the B-field across the western shell for different filling factors $f_{n_{\mathrm{e}}}$.

$E M$, which stems from the $\lambda 6 \mathrm{~cm}$ total intensity measurements. Sun et al. (2007) quote the maximum uncertainty of $10 \%$ for the $\lambda 6 \mathrm{~cm}$ survey data. Besides the uncertainty of $E M$, the errors in $B_{/ /}$also depend on the uncertainties in RM. The standard deviations used for this purpose were listed in Table 2.

We showed the $n_{\mathrm{e}}$ and $B_{/ /}$profiles across the western shell of the W4 superbubble in Fig. 7. Owing to the $1 / \sqrt{f_{n_{\mathrm{e}}}}$ dependence, $f_{n_{\mathrm{e}}}=0.1$ results in high electron density and a large B field, while the values for $f_{n_{\mathrm{e}}}=0.4,0.7$, and 1.0 are much smaller. To distinguish which case is more realistic, we made a simple estimate according to the magnetic flux conservation. For the W4 superbubble, the swept-up magnetic field should be a factor of about 5 higher than the undisturbed Galactic B field when we consider a ring with an inner radius of $65 \mathrm{pc}$ and an outer radius of 72 pc. Using RM data from pulsars, Han et al. (2006) find that the strength of the large-scale regular radial B field of the Galaxy decreases from the inner Galaxy $(4 \mu \mathrm{G})$ to the solar neighbourhood $(2 \mu \mathrm{G})$. If a turbulent field of the same order is considered, the field strength in the W4 superbubble area is about $3 \mu \mathrm{G}$. The 3-D simulation of Sun et al. (2008) and Sun $\&$ Reich (2009) where both regular and turbulent B fields were included gave a similar value of $B_{\text {tot }}$ of around $2.4 \mu \mathrm{G}$. Thus the enhanced line-of-sight B-field strength in the western shell should not exceed $15 \mu \mathrm{G}$ by much, otherwise an amplification mechanism is needed to explain a larger B field for $f_{n_{\mathrm{e}}}=0.1$. If a B-field amplification can be excluded, the filling factor $f_{n_{\mathrm{e}}}$ in the western shell of the W4 superbubble is likely within the range of 0.4 to 1.0. We limit the following discussion to the case that the filling factor $f_{n_{\mathrm{e}}}$ is $0.4,0.7$, and 1.0 , where $f_{n_{\mathrm{e}}}=1.0$ gives the lower limit of the electron density $n_{\mathrm{e}}$ and the strength of the line-of-sight component of the magnetic field $B_{/ /}$. When considering that the line-of-sight passes solely through the western shell (13th to 16th column), we obtained the average $B_{/ /}$to be $-5.0( \pm 10 \%) \mu \mathrm{G}\left(f_{n_{\mathrm{e}}}=1.0\right)$ to $-8.0( \pm 10 \%) \mu \mathrm{G}\left(f_{n_{\mathrm{e}}}=0.4\right)$, and the electron density $n_{\mathrm{e}}$ is between $1.0( \pm 5 \%) \mathrm{cm}^{-3}\left(f_{n_{\mathrm{e}}}=1.0\right)$ and $1.5( \pm 5 \%) \mathrm{cm}^{-3}\left(f_{n_{\mathrm{e}}}=0.4\right)$.

West et al. (2007) used a different analysis method to derive the line-of-sight component of the magnetic field strength in the shell of the W4 supperbubble. Based on the observed depolarization amount, they estimated the PA difference from adjacent lines passing through the shell. From the Faraday screen model, we found the RMs within the shell for the 13th to 16th column between $-70 \mathrm{rad} \mathrm{m}^{-2}$ and $-300 \mathrm{rad} \mathrm{m}^{-2}$, which causes one to four full rotations of PAs at $\lambda 21 \mathrm{~cm}$. West et al. (2007) quote the $\lambda 21 \mathrm{~cm}$ differential angle of $60^{\circ}$ and calculated a line-of-sight B-field strength between $3.4 \mu \mathrm{G}$ and $9.1 \mu \mathrm{G}$ from the different path lengths through the shell. The polarization intensity, polarization angles, and their differences are, however, influenced by the large-scale polarized emission, which is incompletely observed by the DRAO interferometer. Sun et al. (2007) quote the observed Stokes parameter $U, Q$ from a passive Faraday screen as $U=\mathrm{PI}_{\mathrm{fg}} \sin 2 \psi_{\mathrm{fg}}+f \mathrm{PI}_{\mathrm{bg}} \sin \left(2 \psi_{\mathrm{bg}}+2 \psi_{\mathrm{s}}\right)$, $Q=\mathrm{PI}_{\mathrm{fg}} \cos 2 \psi_{\mathrm{fg}}+f \mathrm{PI}_{\mathrm{bg}} \cos \left(2 \psi_{\mathrm{bg}}+2 \psi_{\mathrm{s}}\right)$, where fg and bg denote foreground and background components of PI and PA. Here, $f$ is the depolarization factor and $\psi_{\mathrm{s}}$ is the Faraday rotation angle within the screen. For the W4 superbubble, the foreground and background PA angles are close to zero (see Sect. 4.1.), then $U$ and $Q$ were calculated as $U=f \mathrm{PI}_{\mathrm{bg}} \sin 2 \psi_{\mathrm{s}}$ and $Q=\mathrm{PI}_{\mathrm{fg}}+f \mathrm{PI}_{\mathrm{bg}} \cos 2 \psi_{\mathrm{s}}$ (Eq. (2)). Therefore, the observed depolarization amount in PI and the PA differences for neighbouring lines through the shell depend not only on the difference in $\psi_{\mathrm{s}}$, which is needed for a B-field calculation, but also on $\mathrm{PI}_{\mathrm{fg}}$ and $\mathrm{PI}_{\mathrm{bg}}$. Without taking this into account, the B-field result depends in some way on the large-scale polarization fraction included in the data. Only if the Faraday screen is very local and $\mathrm{PI}_{\mathrm{fg}}=0 \mathrm{mK} T_{\mathrm{b}}$ does this method give the correct results. However, this is not the case for the W4 superbubble.

\subsection{The north-eastern extension}

For better understanding of the magnetic field configuration and the strength of the W4 superbubble, we searched for more parts of shells and arcs, where Faraday effects could be studied in terms of the described Faraday screen model. We note that it is difficult to find unconfused areas with good quality "on" and "off" data. However, a section of the north-eastern extension located above the major eastern shell is noted in the $\lambda 11 \mathrm{~cm}$ polarization image (see Fig. 8), which we found to be suitable for modelling. The $\lambda 6 \mathrm{~cm}$ data show no evidence of depolarization in this region, indicating that the RM is smaller than in the western part of the W4 superbubble. We again applied the Faraday screen model and obtained $f \sim 0.87, c \sim 0.86$ and a positive 

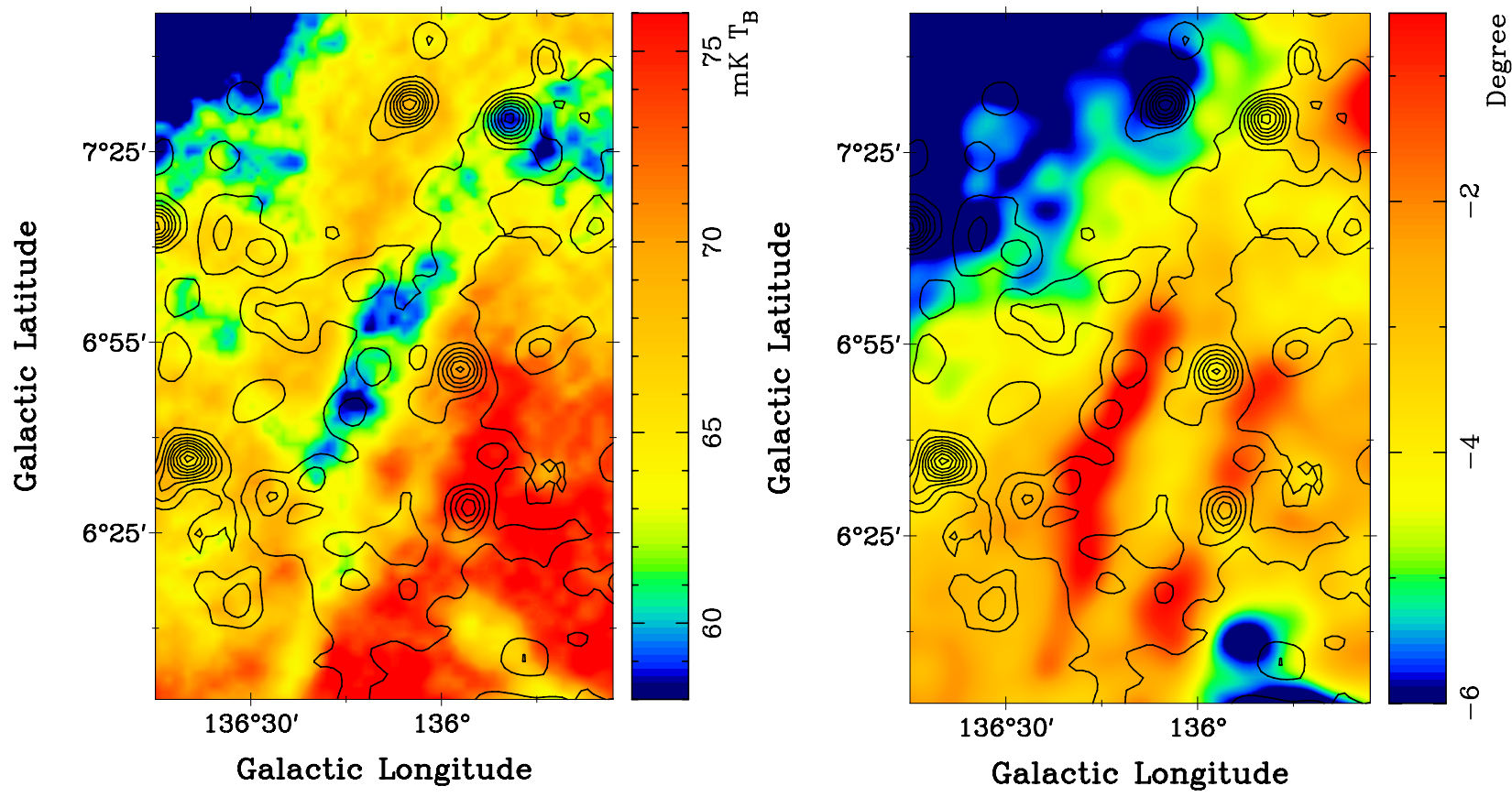

Fig. 8. $\lambda 11 \mathrm{~cm}$ PI (left panel) and PA (right panel) of the north-eastern shell extension overlaid by total intensity contours. The contour levels and the angular resolution of the images are the same as in Fig. 1.

$\mathrm{RM}$ of about $55 \mathrm{rad} \mathrm{m}^{-2}$ in this area. The fitted parameters predict $\mathrm{PI}_{\mathrm{on}} / \mathrm{PI}_{\text {off }}=0.97$ and $\mathrm{PA}_{\text {on }}-\mathrm{PA}_{\text {off }}=1.5$ for the $\lambda 6 \mathrm{~cm}$, and $\mathrm{PI}_{\text {on }} / \mathrm{PI}_{\text {off }}=0.89$ and $\mathrm{PA}_{\text {on }}-\mathrm{PA}_{\text {off }}=-3.9$ for the $\lambda 21 \mathrm{~cm}$ observations, which generally agrees with the non-detection of noticeable Faraday rotated structures in both maps. Following the procedures introduced above, we obtained a lower electron density of $n_{\mathrm{e}}=0.5\left(f_{n_{\mathrm{e}}}=1.0\right)-0.9 \mathrm{~cm}^{-3}\left(f_{n_{\mathrm{e}}}=0.4\right)$ with $1 \sigma$ error of $15 \%$, and a weaker line-of-sight component of the magnetic field $B_{/ /}=3.1\left(f_{n_{\mathrm{e}}}=1.0\right)-4.9 \mu \mathrm{G}\left(f_{n_{\mathrm{e}}}=0.4\right)$ with $1 \sigma$ error of $30 \%$ within the upper north-eastern shell extension.

\section{Discussion and summary}

We have shown that strong regular magnetic fields exist in the W4 superbubble's shells. A study of the process of creating this magnetic field configuration is beyond the scope of this paper, while numerical simulations (e.g. Tomisaka 1998; de Avillez \& Breitschwerdt 2005) provide hints of an evolutionary scenario. To explain the observations, it seems most likely that the originally plane-parallel magnetic fields were lifted up and compressed during the expansion of the W4 superbubble.

The calculation of the total magnetic field strength in the W4 superbubble shell depends on its orientation relative to the line of sight. From the discovery of atomic hydrogen associated to the W4 superbubble, Normandeau et al. (1996) concluded that the W4 superbubble is inclined towards the observer. Lagrois \& Joncas (2009) studied this inclination in detail and found that the inclination angle is between $9^{\circ}$ and $27^{\circ}$. In addition, we measured an inclination angle of $18^{\circ}$ with respect to the Galactic north pole for the western shell (see Fig. 1). If the magnetic field in the W4 superbubble is directed along its shell and if taking its geometry into account, the deprojected regular $B_{\text {tot }}$ field in the western shell can be deduced as $B_{\text {tot }}=B_{/ /} \sqrt{1+\left(\frac{1}{\tan \theta \cos 18^{\circ}}\right)^{2}}=(2.3-6.7) B_{/ /}$, where $\theta$ is the inclination angle between $9^{\circ}$ and $27^{\circ}$.
Thermal and magnetic pressure, besides gravity, ram pressure, and turbulence are key input parameters for superbubble evolution simulations. Thermal pressure is defined as $P_{\text {ther }}=$ $2 n_{0} k T_{\mathrm{e}}$, where $n_{0}=n_{\mathrm{e}}$ for total ionization, $k$ is the Boltzmann constant, and $T_{\mathrm{e}}$ is $8000 \mathrm{~K}$ as assumed above. Magnetic pressure is calculated as $P_{\mathrm{mag}}=B_{\mathrm{tot}}^{2} / 8 \pi$. For each column within the western shell of the W4 superbubble shell (13th, 14th, and 16 th columns, the 15 th column is excluded because of the poor constraints on the B-field), we calculated the thermal and magnetic pressure based on the results shown in Fig. 7 and made a comparison in Table 3. For the inclination angle of $\theta=27^{\circ}$, the magnetic pressure $P_{\text {mag }}$ is generally several times of the thermal pressure $P_{\text {ther }}$, and $P_{\text {mag }}$ becomes overwhelming when the inclination angle $\theta$ approaches to $9^{\circ}$.

For the north-eastern extension in the higher latitude region, $P_{\text {mag }}$ and $P_{\text {ther }}$ are comparable when $\theta \sim 27^{\circ}$, regardless of $f_{n_{\mathrm{e}}}=0.4$ or 1.0. $P_{\text {mag }}$ dominates $P_{\text {ther }}$ if $\theta$ approaches $9^{\circ}$, even for $f_{n_{\mathrm{e}}}=1.0$. A more precise inclination angle would help to better constrain the physical properties of the W4 superbubble.

We found a positive RM on the eastern side and a negative $\mathrm{RM}$ on the western side of the W4 superbubble. This is expected for the scenario described above, where the W4 superbubble expands and breaks out of the Galactic plane and lifts up the magnetic field, which runs clockwise (viewed from the north Galactic pole) in the Perseus arm (Han et al. 2006). Then, for the eastern shell, the field lines will go up and for the western shell downwards. Because the W4 superbubble tilts towards us (Normandeau et al. 1996; Lagrois \& Joncas 2009), the line-ofsight component of the magnetic field points away in the western shell of the W4 superbubble, resulting in a negative RM, and towards us in the eastern shell, where a positive RM is observed.

Radio continuum and polarization observations at $\lambda 6 \mathrm{~cm}$, $\lambda 11 \mathrm{~cm}$, and $\lambda 21 \mathrm{~cm}$ have been made to study the radio emission properties and to estimate the magnetic fields of the W4 superbubble. With the flat radio continuum spectrum found between $\lambda 6 \mathrm{~cm}, \lambda 11 \mathrm{~cm}$, and $\lambda 21 \mathrm{~cm}$, we confirm the thermal origin of 
Table 3. Thermal pressure versus magnetic pressure in the western shell of the W4 superbubble for different filling factors $f_{n_{\mathrm{e}}}$ and the inclination angles of $\theta=27^{\circ}, \theta=18^{\circ}$, and $\theta=9^{\circ}$.

\begin{tabular}{rcccc}
\hline \hline Column No. & $\begin{array}{c}P_{\text {ther }} \\
\left(10^{-12} \mathrm{dyn} \mathrm{cm} \mathrm{cm}^{-2}\right)\end{array}$ & $\begin{array}{c}P_{\mathrm{mag}}, \theta=27^{\circ} \\
\left(10^{-12} \mathrm{dyn} \mathrm{\textrm {cm } ^ { - 2 } )}\right.\end{array}$ & $\begin{array}{c}P_{\mathrm{mag}}, \theta=18^{\circ} \\
\left(10^{-12} \mathrm{dyn} \mathrm{cm}^{-2}\right)\end{array}$ & $\begin{array}{c}P_{\mathrm{mag}}, \theta=9^{\circ} \\
\left(10^{-12} \mathrm{dyn} \mathrm{cm}^{-2}\right)\end{array}$ \\
\hline$f_{n_{\mathrm{e}}}=0.4$ & & & & \\
13 th & $3.6 \pm 0.2$ & $17.5 \pm 3.7$ & $38.2 \pm 8.1$ & $150.0 \pm 31.9$ \\
14 th & $3.4 \pm 0.2$ & $26.3 \pm 7.1$ & $57.4 \pm 15.4$ & $225.5 \pm 60.5$ \\
16 th & $3.0 \pm 0.2$ & $10.3 \pm 2.9$ & $22.4 \pm 6.3$ & $88.1 \pm 24.6$ \\
\hline$f_{n_{\mathrm{e}}}=0.7$ & & & & \\
13 th & $2.7 \pm 0.1$ & $10.0 \pm 2.1$ & $21.8 \pm 4.6$ & $85.7 \pm 18.2$ \\
14 th & $2.6 \pm 0.1$ & $15.0 \pm 4.0$ & $32.8 \pm 8.8$ & $128.9 \pm 34.6$ \\
16 th & $2.3 \pm 0.1$ & $5.9 \pm 1.6$ & $12.8 \pm 3.6$ & $50.3 \pm 14.1$ \\
\hline$f_{n_{\mathrm{e}}}=1.0$ & & & & \\
13 th & $2.3 \pm 0.1$ & $7.0 \pm 1.5$ & $15.3 \pm 3.2$ & $60.0 \pm 12.7$ \\
14 th & $2.1 \pm 0.1$ & $10.5 \pm 2.8$ & $23.0 \pm 6.2$ & $90.2 \pm 24.2$ \\
16 th & $1.9 \pm 0.1$ & $4.1 \pm 1.1$ & $9.0 \pm 2.5$ & $35.2 \pm 9.8$ \\
\hline
\end{tabular}

the radio continuum emission of the $\mathrm{W} 4$ superbubble. Polarized emission shows dramatically morphological differences from wavelength to wavelength. With the advantage of being less affected by Faraday rotation, the $\lambda 6 \mathrm{~cm}$ and $\lambda 11 \mathrm{~cm}$ polarization data were used for the estimates of the line-of-sight component of the magnetic field within the western shell of the W4 superbubble by a passive Faraday screen model. Considering the thermal electron filling factor $f_{n_{\mathrm{e}}}$, the radio continuum observations and a simple geometric assumption result in an electron density of $n_{\mathrm{e}} \sim 1.0 \times 1 / \sqrt{f_{n_{\mathrm{e}}}} \mathrm{cm}^{-3}$, and the line-of-sight component of the magnetic field $B_{/ /} \sim-5.0 \times 1 / \sqrt{f_{n_{\mathrm{e}}}} \mu \mathrm{G}$ (pointing away from us) for the western shell of the W4 superbubble, where the typical error is about $5 \%$ for $n_{\mathrm{e}}$ and $10 \%$ for $B_{/ /}$. Based on a simple estimate, we found that $f_{n_{\mathrm{e}}}$ likely has a value greater than 0.1 assumed for H II regions (Harvey-Smith et al. 2011). Being related to the inclination angle of the superbubble with respect to the plane of the sky, a total magnetic field is found above $12 \mu \mathrm{G}$. This results in a magnetic pressure, which is one or two orders of magnitude higher than the thermal pressure in the western shell of the W4 superbubble. The $\lambda 11 \mathrm{~cm}$ polarization data allow a model fit of the weaker RM in the high-latitude north-eastern shell, where we find a lower electron density of $n_{\mathrm{e}}=0.5$ to $0.9 \mathrm{~cm}^{-3}$ and $B_{/ /}$of $3.1-4.9 \mu \mathrm{G}$ for filling factors $f_{n_{\mathrm{e}}}$ of 1.0 or 0.4 , respectively. This means that the magnetic and thermal pressure might be comparable in the upper parts of the superbubble. The RM sign reverses as expected for a scenario where the Galactic magnetic field is pushed out of the plane by the expanding W4 superbubble, which is tilted towards us. These results are expected to constrain magneto-hydrodynamical simulations of the W4 superbubble and superbubbles in general.

Acknowledgements. X.Y.G. and J.L.H. are supported by the National Natural Science foundation of China $(11303035,11473034)$ and the Partner group of the MPIfR at NAOC in the framework of the exchange programme between MPG and CAS for many bilateral visits. X.Y.G. acknowledges financial support by the MPG, by Michael Kramer during his stay at the MPIfR, Bonn, and the Young Researcher Grant of National Astronomical Observatories, Chinese Academy of Sciences. This research is based in part on observations with the Effelsberg $100-\mathrm{m}$ telescope of the MPIfR. We would like to thank the anonymous referee for helpful comments and suggestions.

\section{References}

Basu, S., Johnstone, D., \& Martin, P. G. 1999, ApJ, 516, 843 Bennett, C. L., Larson, D., Weiland, J. L., et al. 2013, ApJS, 208, 20
Brinks, E., \& Bajaja, E. 1986, A\&A, 169, 14

Cash, W., Charles, P., Bowyer, S., et al. 1980, ApJ, 238, L71

Condon, J. J., Cotton, W. D., Greisen, E. W., et al. 1998, AJ, 115, 1693

Crawford, F., \& Tiffany, C. L. 2007, AJ, 134, 1231

de Avillez, M. A., \& Breitschwerdt, D. 2005, A\&A, 436, 585

Dennison, B., Topasna, G. A., \& Simonetti, J. H. 1997, ApJ, 474, L31

Deul, E. R., \& den Hartog, R. H. 1990, A\&A, 229, 362

Ferrière, K. M., Mac Low, M.-M., \& Zweibel, E. G. 1991, ApJ, 375, 239

Finkbeiner, D. P. 2003, ApJS, 146, 407

Gao, X. Y., Reich, W., Han, J. L., et al. 2010, A\&A, 515, A64

Graham, J. A., \& Lawrie, D. G. 1982, ApJ, 253, L73

Haffner, L. M., Reynolds, R. J., \& Tufte, S. L. 1998, ApJ, 501, L83

Han, J. L., Manchester, R. N., Lyne, A. G., Qiao, G. J., \& van Straten, W. 2006, ApJ, 642, 868

Harvey-Smith, L., Madsen, G. J., \& Gaensler, B. M. 2011, ApJ, 736, 83

Heiles, C. 1979, ApJ, 229, 533

Heiles, C. 1984, ApJS, 55, 585

Heiles, C. 2000, AJ, 119, 923

Hiltner, W. A. 1956, ApJS, 2, 389

Hinshaw, G., Weiland, J. L., Hill, R. S., et al. 2009, ApJS, 180, 225

Hu, E. M. 1981, ApJ, 248, 119

Kim, S., Staveley-Smith, L., Sault, R. J., et al. 1998, PASA, 15, 132

Komljenovic, P. T., Basu, S., \& Johnstone, D. 1999, in New Perspectives on the Interstellar Medium, eds. A. R. Taylor, T. L. Landecker, \& G. Joncas, ASP Conf. Ser., 168, 299

Koo, B.-C., Heiles, C., \& Reach, W. T. 1992, ApJ, 390, 108

Lagrois, D., \& Joncas, G. 2009, ApJ, 693, 186

Landecker, T. L., Dewdney, P. E., Burgess, T. A., et al. 2000, A\&AS, 145, 509

Landecker, T. L., Reich, W., Reid, R. I., et al. 2010, A\&A, 520, A80

Maciejewski, W., Murphy, E. M., Lockman, F. J., \& Savage, B. D. 1996, ApJ, 469,238

Mac Low, M.-M., \& McCray, R. 1988, ApJ, 324, 776

Mac Low, M.-M., McCray, R., \& Norman, M. L. 1989, ApJ, 337, 141

McClure-Griffiths, N. M., Dickey, J. M., Gaensler, B. M., et al. 2000, AJ, 119, 2828

McClure-Griffiths, N. M., Ford, A., Pisano, D. J., et al. 2006, ApJ, 638, 196

Meaburn, J. 1980, MNRAS, 192, 365

Müller, P., Reif, K., \& Reich, W. 1987, A\&A, 183, 327

Norman, C. A., \& Ikeuchi, S. 1989, ApJ, 345, 372

Normandeau, M., Taylor, A. R., \& Dewdney, P. E. 1996, Nature, 380, 687

Page, L., Hinshaw, G., Komatsu, E., et al. 2007, ApJS, 170, 335

Pidopryhora, Y., Lockman, F. J., \& Shields, J. C. 2007, ApJ, 656, 928

Reich, W. 1982, A\&AS, 48, 219

Reich, W. 2006, in Cosmic Polarization, ed. R. Fabbri (Research signpost), 91

Reich, P., \& Reich, W. 1986, A\&AS, 63, 205

Reich, W., Fürst, E., Reich, P., et al. 2004, in The Magnetized Interstellar

Medium, eds. B. Uyanıker, W. Reich, \& R. Wielebinski (Copernikus), 45

Reynolds, R. J., \& Ogden, P. M. 1979, ApJ, 229, 942

Reynolds, R. J., Sterling, N. C., \& Haffner, L. M. 2001, ApJ, 558, L101

Spoelstra, T. A. T. 1984, A\&A, 135, 238

Stil, J., Wityk, N., Ouyed, R., \& Taylor, A. R. 2009, ApJ, 701, 330

Sun, X. H., \& Reich, W. 2009, A\&A, 507, 1087

Sun, X.-H., \& Reich, W. 2010, Res. Astron. Astrophys., 10, 1287 
Sun, X. H., Han, J. L., Reich, W., et al. 2007, A\&A, 463, 993

Sun, X. H., Reich, W., Waelkens, A., \& Enßlin, T. A. 2008, A\&A, 477, 573

Sun, X. H., Reich, W., Han, J. L., et al. 2011, A\&A, 527, A74

Taylor, A. R., Gibson, S. J., Peracaula, M., et al. 2003, AJ, 125, 3145

Taylor, A. R., Stil, J. M., \& Sunstrum, C. 2009, ApJ, 702, 1230

Terebey, S., Fich, M., Taylor, R., Cao, Y., \& Hancock, T. 2003, ApJ, 590, 906

Tomisaka, K. 1990, ApJ, 361, L5

Tomisaka, K. 1998, MNRAS, 298, 797

Tomisaka, K., \& Ikeuchi, S. 1986, PASJ, 38, 697

Turtle, A. J., Pugh, J. F., Kenderdine, S., \& Pauliny-Toth, I. I. K. 1962, MNRAS 124, 297

Uyanıker, B. 2004, in The Magnetized Interstellar Medium, eds. B. Uyanıker, W. Reich, \& R. Wielebinski (Copernikus), 71

Uyanıker, B., Fürst, E., Reich, W., Reich, P., \& Wielebinski, R. 1998, A\&AS, 132,401
Uyanıker, B., Fürst, E., Reich, W., Reich, P., \& Wielebinski, R. 1999, A\&AS, 138,31

Uyanıker, B., Fürst, E., Reich, W., Aschenbach, B., \& Wielebinski, R. 2001, A\&A, 371, 675

Vallée, J. P. 1982, ApJ, 261, L55

Vallée, J. P. 1993, ApJ, 419, 670

West, J. L., English, J., Normandeau, M., \& Landecker, T. L. 2007, ApJ, 656, 914

Wilson, T. L., Rohlfs, K., \& Hüttemeister, S. 2013, Tools of Radio Astronomy (Berlin: Springer-Verlag)

Wolleben, M., Landecker, T. L., Reich, W., \& Wielebinski, R. 2006, A\&A, 448, 411

Xiao, L., Han, J. L., Reich, W., et al. 2011, A\&A, 529, A15

Xu, J., \& Han, J.-L. 2014, RA\&A, 14, 942

Xu, Y., Reid, M. J., Zheng, X. W., \& Menten, K. M. 2006, Science, 311, 54 